\title{
Aggregated Negative Feedback in a Generalized Lorenz Model
}

\author{
Bo-Wen Shen \\ Department of Mathematics and Statistics, \\ San Diego State University, 5500 Campanile Drive, \\ San Diego, CA 92182, USA \\ bshen@mail.sdsu.edu
}

Received July 25, 2018; Revised September 17, 2018

\begin{abstract}
In this study, we first present a generalized Lorenz model (LM) with $M$ modes, where $M$ is an odd number that is greater than three. The generalized LM (GLM) is derived based on a successive extension of the nonlinear feedback loop (NFL) with additional high wavenumber modes. By performing a linear stability analysis with $\sigma=10$ and $b=8 / 3$, we illustrate that: (1) within the 3D, 5D, and 7D LMs, the appearance of unstable nontrivial critical points requires a larger Rayleigh parameter in a higher-dimensional LM and (2) within the 9DLM, nontrivial critical points are stable. By comparing the GLM with various numbers of modes, we discuss the aggregated negative feedback enabled by the extended NFL and its role in stabilizing solutions in high-dimensional LMs. Our analysis indicates that the 9DLM is the lowest order generalized LM with stable nontrivial critical points for all Rayleigh parameters greater than one. As shown by calculations of the ensemble Lyapunov exponent, the 9DLM still produces chaotic solutions. Within the 9DLM, a larger critical value for the Rayleigh parameter, $r_{c}=679.8$, is required for the onset of chaos as compared to a $r_{c}=24.74$ for the 3DLM, a $r_{c}=42.9$ for the 5DLM, and a $r_{c}=116.9$ for the 7DLM. In association with stable nontrivial critical points that may lead to steady-state solutions, the appearance of chaotic orbits indicates the important role of a saddle point at the origin in producing the sensitive dependence of solutions on initial conditions. The 9DLM displays the coexistence of chaotic and steady-state solutions at moderate Rayleigh parameters and the coexistence of limit cycle and steady-state solutions at large Rayleigh parameters. The first kind of coexistence appears within a smaller range of Rayleigh parameters in lower-dimensional LMs (i.e. $24.06<r<24.74$ within the 3DLM) but in a wider range of Rayleigh parameters within the 9DLM (i.e. $679.8<r<1058$ ). The second kind of coexistence has never been reported in high-dimensional Lorenz systems.
\end{abstract}

Keywords: Chaos; Lorenz model; nonlinear feedback loop; nonlinear negative feedback.

\section{Introduction}

Our current view on the predictability of weather and climate has been significantly influenced by the model of Prof. Lorenz of MIT [Lorenz, 1963]. The Lorenz model represents an elegant set of three ordinary differential equations (ODEs) that are based on a system of partial differential equations (PDEs) for the Rayleigh-Benard convection (RBC) [e.g. Saltzman, 1962]. The Lorenz model with three variables is referred to as the 3DLM. The complexities of solutions in the temporal space have a profound impact on our understanding of the

This is an Open Access article published by World Scientific Publishing Company. It is distributed under the terms of the Creative Commons Attribution 4.0 (CC-BY) License. Further distribution of this work is permitted, provided the original work is properly cited. 
dynamics of nonlinear systems investigated in many fields (e.g. Gleick, 1987]). One type of solution appears irregularly oscillatory and is highly sensitive to initial conditions. Two initial nearby orbits diverge over time but remain bounded. These solutions are now known as chaotic solutions and their characteristics are called the butterfly effect (e.g. Gleick, 1987; Lorenz, 1993, 2008; Solomon et al., 2007; Anthes, 2011]). Recently, the sensitive dependence on initial conditions has been referred to as the butterfly effect of the first kind [BE1, Shen, 2014]. In comparison, its interpreted meaning is a metaphor for indicating that a tiny perturbation may create an organized large-scale system (e.g. Lorenz, 1969, 1972 as the butterfly effect of the second kind [BE2, Shen, 2014; Shen et al., 2018]).

Chaotic solutions appear in nonlinear systems. Therefore, as discussed below, numerous studies have been performed for examining the role of nonlinearity in producing chaotic responses and for revealing the source of chaos as well as the source of recurrence. To achieve these goals, high-dimensional Lorenz models (LMs) have been developed (e.g. Currv, 1978; Currv et al., 1984; Howard \& Krishnamurti, 1986; Hermiz et al., 1995; Thiffeault \& Horton, 1996; Musielak et al., 2005; Rov \& Musielak, 2007a. 2007b. 2007d; Shen, 2014, 2015, 2016, 2017: Moon et al., 2017: Felicio \& Rech, 2018]). As a result of the increased complexities of the Jacobian term in the RBC equations using additional spatial Fourier modes, the degree of nonlinearity is increased in higher-dimensional LMs. However, "high-dimensional LMs" still have a limited degree of nonlinearity. In 1996 and 2005, Lorenz proposed different chaotic systems using many modes [Lorenz, 1996, 2005], but such systems are not derived from physically-based PDEs. The validity of the Lorenz [1996/2005] models in realworld applications is less known. Therefore, an idealized model with a sufficient number of modes is still desirable for improving our understanding of the chaotic nature of weather, as well as the role of chaos in numerical weather prediction (NWP). As previously mentioned, the original Lorenz 1963 model was derived from the RBC equations. Therefore, in this study, we extend our recent studies (e.g. Shen, 2014, 2016, 2017]) in order to generalize the Lorenz 1963 model using many modes to study the impact of an increased degree of nonlinearity, as well as its collective effect with heating and dissipations, on solution stability. Our goals are to improve the representation of physical processes in real-world models (e.g. Shen et al., 2006, 2010, 2013]), to improve NWPs, and to understand the nature of weather.

While high-dimensional LMs with many modes are required to solve real-world problems, simplified models can be used to understand the fundamental dynamics of Lorenz's chaotic solutions. Table 1 lists three of the simplified models, discussed below. The so-called geometric model (e.g. [Guckenheimer \& Williams, 1979; Hirsch et al., 2013]) consists of

Table 1. The Lorenz model Lorenz, 1963] and three simplified versions, including the geometric model Guckenheimer \& Williams, 1979], the limiting equations [Sparrow, 1982], and the nondissipative Lorenz model [Shen, 2018].

(1) The Lorenz Model

$$
\begin{aligned}
& \frac{d X}{d \tau}=-\sigma X+\sigma Y \\
& \frac{d Y}{d \tau}=-X Z+r X-Y, \\
& \frac{d Z}{d \tau}=X Y-b Z .
\end{aligned}
$$

(3) The Limiting Equations

$$
\begin{aligned}
& \frac{d X}{d \tau}=\sigma Y \\
& \frac{d Y}{d \tau}=-X Z \\
& \frac{d Z}{d \tau}=X Y
\end{aligned}
$$

(2) The Geometric Model

$$
\begin{aligned}
& \frac{d X}{d \tau}=-3 X \\
& \frac{d Y}{d \tau}=2 Y, \\
& \frac{d Z}{d \tau}=-Z .
\end{aligned}
$$

(4) The Nondissipative Lorenz Model

$$
\begin{aligned}
& \frac{d X}{d \tau}=\sigma Y \\
& \frac{d Y}{d \tau}=-X Z+r X \\
& \frac{d Z}{d \tau}=X Y .
\end{aligned}
$$


three uncoupled linear ODEs with one critical point at the origin and three real eigenvalues, including two negative values and one positive value. Since a positive eigenvalue leads to a constant growth rate, the corresponding solutions are unbounded. Therefore, additional assumptions, such as returning conditions, were made [Hirsch et al., 2013]. To understand how accurately the geometric model describes the dynamics of the strange attractor within the Lorenz model, the 14th mathematical problem of Smale's list [Smale, 1998] looked for a proof (i.e. homeomorphism) for revealing dynamic equivalence between the 3DLM and the geometric model. To illustrate the dynamic similarity of solutions between the 3DLM and the geometric model of Guckenheimer and Williams, a rigorous proof was provided by Tucker 2002]. Tucker's [2002] study suggests that the Lorenz strange attractor is not a numerical artifact Stewart, 2000]. Since the geometric model only has one critical point as a saddle point at the origin, Tucker's 2002] work supports the view that the saddle point is one of the essential ingredients for sensitive dependence on initial conditions (ICs). While the assumption of the "returning condition" in the geometric model may be responsible for the nature of irregular oscillations for chaotic solutions, as justification for the assumption, identifying one or more terms within the 3DLM is required.

Using 3D and 5D nondissipative Lorenz models (NLMs), we have recently studied the role of nonlinear terms in producing recurrence Shen, 2018; Shen \& Faghih-Naini, 2017; Faghih-Naini \& Shen, 2018]. Mathematically, two nonlinear terms within the 3DLM [e.g. $-X Y$ and $X Z$ in Eqs. (2) and (3)] form a nonlinear feedback loop (NFL). The 3DNLM can be reduced to a special form of the Duffing equation [Bender \& Orszag, 1978], and the NFL leads to a cubic term that acts as a nonlinear restoring force for producing nonlinear oscillatory solutions Shen, 2018. The NFL is also responsible for the appearance of homoclinic orbits that connect the stable and unstable manifolds of a saddle point at the origin. By comparing earlier studies, we found that the 3D-NLM with no heating term is identical to the simplified set of the Lorenz model, the socalled limiting equations (e.g. Eq. (2) in Sparrow, 1982, p. 135]), that was used to reveal the periodic nature of limit cycle (LC) solutions for large Rayleigh parameters (as discussed in Sec. 2). The LC is defined as an isolated closed orbit and nearby trajectories spiral into the closed orbit Sprott, 2003; Jordan \& Smith, 2007; Strogatz, 2015]. More interestingly, LC orbits are determined by the structure of the system itself (i.e. independent of initial conditions). By extending the 3D-NLM, FaghihNaini and Shen 2018] found that the linearized 5D-NLM produces two incommensurate frequencies whose ratio is irrational, leading to quasi-periodic solutions. This type of solution appears "recurrent" but never repeats. Additionally, Faghih-Naini and Shen 2018] provided a mathematical analogy between the linearized 5D-NLM and a system with two springs and two masses. The analogy reveals how an extension of the NFL with the 5DLM couples two systems, each with one commensurate frequency, into a composite system that possesses two incommensurate frequencies. Therefore, the NFL plays a role in transferring energy across two spatial scales and in creating incommensurate frequencies. Studies with NLMs suggest that the returning conditions of the geometric model are related to the NFL. A challenging question of whether and how the aforementioned features may exist in higherdimensional LMs is addressed in this study.

Our paper is organized as follows. In Sec. 2, we present a GLM with $M$ modes, where $M$ is an odd number that is greater than three. Conservative quantities under dissipationless conditions are derived, as part of verification for the selection of Fourier modes. The special case of $M=9$, leading to the 9DLM, is discussed. The new 9DLM presented here is different from the 9DLM discussed in Shen, 2017. In Sec. 3, we first discuss how solutions of nontrivial critical points for the 9DLM are obtained. We then define a nonlinear feedback term and represent it using solutions of the nontrivial critical points to reveal the aggregated negative feedback that is associated with successive extension of the NFL within the GLM. By applying the critical point solution in order to linearize the 9DLM for the linear stability analysis, we then illustrate that the 9DLM represents a minimal set of ODEs that possess stable, nontrivial critical points for all Rayleigh parameters greater than one when typical values are used for remaining parameters such as $\sigma=10$ and $b=8 / 3$. We additionally calculate the Lyapunov exponents Wolf et al., 1985. as a function of the Rayleigh parameters and perform numerical simulations in order to illustrate (1) the coexistence of chaotic and nonchaotic solutions and (2) the coexistence of the limit cycle and 
steady-state solutions. Concluding remarks are provided at the end. In Appendix A, we provide a mathematical analogy between the linearized 9D nondissipative LM and a coupled system with four masses and four springs.

\section{Lorenz Models}

\subsection{A generalized $M$-dimensional Lorenz model}

Based on the same approach used in Shen, 2014] that extends the NFL of the 3DLM using additional modes, we derive the following generalized Lorenz model (GLM) with $M$ modes ( $M \geq 5$ is an odd number):

$$
\begin{aligned}
\frac{d X}{d \tau}= & -\sigma X+\sigma Y, \\
\frac{d Y}{d \tau}= & -X Z+r X-Y, \\
\frac{d Z}{d \tau}= & X Y\left(-X Y_{1}-b Z,\right. \\
\frac{d Y_{j}}{d \tau}= & j X Z_{j-1}-(j+1) X Z_{j} \\
& -d_{j-1} Y_{j}, \quad j \in[1, N], \\
\frac{d Z_{j}}{d \tau}= & (j+1) X Y_{j}-(j+1) X Y_{j+1} \\
& -\beta_{j} Z_{j}, \quad j \in[1, N], \\
N=\frac{M-3}{2} ; \quad d_{j-1}=\frac{(2 j+1)^{2}+a^{2}}{1+a^{2}} ; & \beta_{j}=(j+1)^{2} b .
\end{aligned}
$$

Here, $\tau=\kappa\left(1+a^{2}\right)(\pi / H)^{2} t$ (dimensionless time) and $t$ represents time. The constant $\kappa$ denotes the thermal conductivity. The parameter $a$ is defined as the ratio of the vertical scale of the convection cell to its horizontal scale. The term $H$ is the domain height, and $2 \mathrm{H} / \mathrm{a}$ indicates the domain width. $\sigma=\nu / \kappa$ is the Prandtl number and $\nu$ represents the kinematic viscosity. $r=R_{a} / R_{c}$ is the normalized Rayleigh number, or the heating parameter. $R_{a}$ is the Rayleigh number and $R_{c}$ is its critical value for the free-slip Rayleigh-Benard problem. Both $R_{a}$ and $R_{c}$ are defined in Eq. (9) of Shen, 2014. For convenience, $r$ is also referred to as the Rayleigh parameter. $a^{2}=1 / 2$ and $b=4 /\left(1+a^{2}\right)=$ 8/3. Additionally, $Z_{0}=Z$ in Eq. (4) and $Y_{N+1}=0$ in Eq. (5). For the special case of $N=0$ associated with $M=3, Y_{1}=0$ and Eqs. (1)-(3) are the 3DLM. Similarly, when $M=5,7$, and 9, Eqs. (10)-(6) represent the 5D, 7D, and 9DLMs, respectively.

The GLM was derived based on an extension of the NFL using high wavenumber modes for temperature, leading to additional ODEs that describe the time evolution of $Y_{j}$ and $Z_{j}$ with $j \in[1, N]$. Since the index $j$ is proportional to the wavenumber, a mode with a larger $j$ represents a smaller scale mode. Given a pair of $(j)$ th modes (e.g. $\left(Y_{j}, Z_{j}\right)$ ), the time evolution is described in Eqs. (44) and (5), where terms with a subscript of $j-1$ (i.e. $X Z_{j-1}$ ) and $j+1$ (i.e. $X Y_{j+1}$ ) represent an upscale (downscale) transfer to (from) the $(j-1)$ th mode and a downscale (upscale) transfer to (from) the $(j+1)$ th mode, respectively. Within the pair for the $(j)$ th mode, $-X Z_{j}$ and $X Y_{j}$ form a small feedback loop. As a result, the original nonlinear feedback (i.e. $X Z$ and $X Y)$ is successively extended to include downscale transfer to the $(j+1)$ th mode via $X Y_{j+1}$. Thus, the extended NFL provides successive feedback from the $(j+1)$ th mode via the $X Y_{j+1}$, as well.

While the GLM applies additional high wavenumber modes to represent the temperature field, the original PDEs for the Rayleigh-Benard convection Saltzman, 1962] describe the time evolution of both the streamfunction and temperature. Justifications are provided below. When $M=5$ or 7 , the GLM represents the 5DLM [Shen, 2014] or 7DLM [Shen, 2016], respectively. For temperature modes within the 7DLM, we previously referred to $(Y, Z),\left(Y_{1}, Z_{1}\right)$, and $\left(Y_{2}, Z_{2}\right)$ as the primary, secondary, and tertiary modes, respectively. In comparison, for the streamfunction, the GLM only contains the primary mode (i.e. $X$ ). The impact of the secondary and tertiary modes for the streamfunction was previously examined by comparing the 5DLM and 6DLM [Shen, 2015] and by comparing the 7DLM and an alternative 9DLM [Shen, 2017], where the 6DLM includes a secondary mode and the 9DLM contains additional secondary and tertiary modes. Streamfunction modes at high wavenumbers have been found to introduce additional heating terms and provide positive feedback. However, due to scale asymmetry, high wavenumber streamfunction modes, in general, have small amplitudes. Their impacts are less important, with the exception of their role in introducing additional heating terms and additional critical points. Derivations of the 
extended NFL within the 5DLM and 6DLM were documented in the supplemental materials of Shen, 2015]. As a result, within the GLM, we focus on the thermodynamic negative feedback associated with additional high wavenumber modes for temperature. In the following, we discuss the aggregated negative feedback by various small scale modes and illustrate the role of aggregated negative feedback in producing stable nontrivial critical points and, thus, the coexistence of chaotic and nonchaotic solutions within a new 9DLM (i.e. the GLM with $M=9$ ).

\subsection{Energy conservation under the dissipationless condition}

Within the GLM in Eqs. (1)-(6), dissipative terms appear as linear terms with a negative sign. Below, by ignoring them, we show that the system contains conservative quantities. Using Eqs. (14)-(16) of [Shen, 2015] for the definitions of domain-averaged kinetic energy $(\overline{\mathrm{KE}})$, available potential energy $(\overline{\mathrm{APE}})$, and potential energy $(\overline{\mathrm{PE}})$. [Treve \& Manley, 1982: Thiffeault \& Horton. 1996: Blender \& Lucarini, 2013; Shen, 2014], we obtain the following equations:

$$
\begin{aligned}
\overline{\mathrm{KE}} & =\frac{C_{o}}{2}\left(X^{2}\right), \\
\overline{\mathrm{APE}} & =-\frac{C_{o}}{2} \frac{\sigma}{r} \sum_{j=0}^{N}\left(Y_{j}^{2}+Z_{j}^{2}\right), \\
\overline{\mathrm{PE}} & =-C_{o} \sigma \sum_{j=0}^{N}\left(\frac{Z_{j}}{j+1}\right),
\end{aligned}
$$

here, $C_{o}=\pi^{2} \kappa^{2}\left(\frac{1+a^{2}}{a}\right)^{3}$. Simply speaking, the above equations (7)-(9) represent the $\overline{\mathrm{KE}}, \overline{\mathrm{PE}}$, and $\overline{\mathrm{APE}}$, respectively, in the phase space within the 9DLM. Equations (17) and (8) yield the following:

$\overline{\mathrm{KE}}+\overline{\mathrm{APE}}=\frac{C_{o}}{2}\left(X^{2}-\frac{\sigma}{r} \sum_{j=0}^{N}\left(Y_{j}^{2}+Z_{j}^{2}\right)\right)=C_{3}$,

while Eqs. (7) and (9) lead to the following:

$$
\overline{\mathrm{KE}}+\overline{\mathrm{PE}}=C_{o}\left(\frac{X^{2}}{2}-\sigma\left(\sum_{j=0}^{N} \frac{Z_{j}}{j+1}\right)\right)=C_{4} .
$$

With Eqs. (1)-(6) in the dissipationless limit, the time derivative of both Eqs. (10) and (11) are zero, so both $C_{3}$ and $C_{4}$ are constants. Therefore, Eqs. (10) and (11) indicate two energy conservation laws. For the special case with $N=0$ (i.e. the 3DLM), the two conservative quantities in Eqs. (10) and (11) are related to the Nambu Hamiltonians Nambu, 1973; Nevir \& Blender, 1994; Floratos, 2012; Roupas, 2012; Blender \& Lucarini, 2013.

Below, we discuss the "various forms of nonlinearity", including mode-mode interactions within the physical spatial space and perturbationperturbation interactions within the temporal space. Given the selected Fourier modes, the nonlinear 3DLM shows the complexity of solutions within the temporal space. Solution complexity is further enhanced by additional nonlinear terms within high-dimensional LMs that allow extra spatial mode-mode interactions via the Jacobian. In other words, the complexity of spatial mode-mode interaction (depending on the choice of Fourier modes) can enhance the complexity of the solution in the temporal space. To better discuss the role of nonlinearity, we use the term interconnectivity to indicate the interconnectedness of various modes at different spatial and temporal scales determined by the NFL and its extensions, including basic state-perturbation interactions and perturbationperturbation interactions. For example, we can increase the degree of interconnectivity within the physical spatial space by adding more spatial Fourier modes. An improved degree of interconnectivity with additional spatial modes is crucial for a tiny perturbation to produce chain reactions across spatial scales. By comparison, for better system tracking, we can decrease the degree of interconnectivity within the time space by linearizing time-varying amplitudes with respect to a (timeindependent) basic state.

Thus, for a set of linearized ODEs (with a linearized NFL), representing the temporal evolution of the amplitude perturbation, interconnectivity is simplified within the temporal space but still reserves scale coupling processes associated with spatial mode-mode interactions. Since a linearized system is mathematically simpler (than its nonlinear version), it is effective for revealing the role of the NFL in transferring energy across scales and in creating incommensurate frequencies. As discussed in Appendix A, we analyze a linearized nondissipative system with nine modes and provide a mathematical analogy with a coupled system that has four masses and springs. We found that each additional 
pair of spatial modes $\left[Y_{j}, Z_{j}\right.$ in Eqs. (4) and (5)] can produce one additional incommensurate frequency. Therefore, the NFL and its extensions with pairs of spatial modes may lead to recurrent solutions with multiple frequencies and, thus, increase the complexities of solutions within the temporal space. However, since interconnectivity is simplified within the temporal space in the linearized system, chaotic solutions cannot exist. Note that the geometric model of Guckenheimer and Williams [1979] is linear and, thus, does not possess recurrent solutions unless an additional assumption such as the returning condition is made.

The aforementioned linearized nondissipative system is effective for revealing the role of the extended NFL in producing additional oscillatory components, as well as transferring energy across scales. When dissipations are considered, the complexity of solutions displays dependence on the collective impact of the extended NFL, dissipative and heating terms. Mathematically, the complexity of solutions over a (short) time period may be indicated by the number of real or complex eigenvalues in a linearized system and the relative magnitude of the real and imaginary parts of the eigenvalues. For example, a composite solution may consist of multiple eigenmodes with multiple incommensurate frequencies that are associated with various imaginary parts of eigenvalues. A specific eigenmode with a large decay rate that is associated with a negative real part of an eigenvalue dissipates quickly, reducing the temporal complexity of a solution over a long period of time.

\subsection{A nine-dimensional Lorenz model (9DLM)}

By analyzing the GLM with various numbers of modes in Eqs. (1) - (6), we illustrate aggregated negative feedback using the 5DLM, 7DLM, and 9DLM, the latter of which is written as follows:

$$
\begin{aligned}
& \frac{d X}{d \tau}=-\sigma X+\sigma Y, \\
& \frac{d Y}{d \tau}=-X Z+r X-Y, \\
& \frac{d Z}{d \tau}=X Y-X Y_{1}-b Z, \\
& \frac{d Y_{1}}{d \tau}=X Z-2 X Z_{1}-d_{o} Y_{1},
\end{aligned}
$$

$$
\begin{aligned}
& \frac{d Z_{1}}{d \tau}=2 X Y_{1}-2 X Y_{2}-4 b Z_{1} \\
& \frac{d Y_{2}}{d \tau}=2 X Z_{1}-3 X Z_{2}-d_{1} Y_{2} \\
& \frac{d Z_{2}}{d \tau}=3 X Y_{2}-3 X Y_{3}-9 b Z_{2} \\
& \frac{d Y_{3}}{d \tau}=3 X Z_{2}-4 X Z_{3}-d_{2} Y_{3} \\
& \frac{d Z_{3}}{d \tau}=4 X Y_{3}-16 b Z_{3}
\end{aligned}
$$

In addition to the primary, secondary, and tertiary modes, the newly-added modes $\left(Y_{3}, Z_{3}\right)$ within the 9DLM are referred to as quaternary modes. The 9DLM is reduced to the 7DLM when quaternary modes are neglected and to the 5DLM when tertiary and quaternary modes are neglected. The 9DLM that ignores the secondary, tertiary, and quaternary modes represents the 3DLM.

Here, we qualitatively discuss the aggregated negative feedback associated with thermal dissipative processes, while a detailed mathematical analysis is provided in Sec. 3.1 Equations (12)-(20) can be viewed as a 3DLM with a feedback process, indicated by $X Y_{1}$ in Eq. (14) with a secondary mode $\left(Y_{1}\right)$ that is governed by Eq. (15) as well as Eq. (16). Furthermore, $X Y_{1}$ represents successive feedbacks from the tertiary modes [i.e. $\left(Y_{2}, Z_{2}\right)$ governed by Eqs. (17) and (18)], whose feedback is obtained from the quaternary modes [i.e. $\left(Y_{3}, Z_{3}\right)$ governed by Eqs. (19) and (20)]. Simply speaking, the $X Y_{1}$ represents an aggregated feedback by the secondary, tertiary, and quaternary modes.

\section{Discussions}

In this section, we first present solutions for the nontrivial critical points of the 9DLM and then apply them to linearize the 9DLM in order to perform a linear stability analysis. We also present the Lyapunov exponent analysis and discuss numerical results using the 9DLM.

\subsection{Solutions of critical points and aggregated feedback}

Within the GLM using various (M) modes, including the 5D, 7D, and 9D LMs, the time derivative for the primary modes [i.e. $d X / d \tau, d Y / d \tau$, and $d Z / d \tau$ in Eqs. (11)-(3)] have the same form. A comparison of Eqs. (1)-(3) of the GLM with the 3DLM 
indicates that the only difference is the appearance of the term $X Y_{1}$ in red, which "explicitly" involves only a secondary mode and is referred to as a feedback term. As discussed below, the major difference among these high-dimensional models is the degree of complexity of the feedback term $X Y_{1}$ in the equation for $d Z / d \tau$ [e.g. Eq. (3) or Eq. (14)]. Thus, by applying a mathematical analysis of $Y_{1}$ using critical point solutions within the 5D, 7D, and 9D LMs, we illustrate the "aggregated nature" of negative feedback through the $X Y_{1}$.

After lengthy derivations, we obtain the following single equation for the critical points of the 9DLM:

$$
\begin{gathered}
X^{2}-X Y_{1}-b Z=0 \\
Y_{1}=\frac{b X Z T_{1}}{X^{2}+T_{2}}, \\
T_{1}=\frac{3 X^{4}+2 b X^{2}\left(d_{1}+d_{2}\right)+b^{2} d_{1} d_{2}}{X^{4}+\left(b d_{2}+2 b d_{1}\right) X^{2}+b^{2} d_{1} d_{2}}, \\
T_{2}=b d_{o} T_{1} .
\end{gathered}
$$

$T_{1}$ and $T_{2}$ in Eqs. (23) are functions of $X$, so $Y_{1}$ in Eq. (22) is also a function of $X$. Since critical point solutions for $Z$ can be obtained from Eqs. (1) and (2) [or Eqs. (12) and (13)], leading to $Z_{c}=0$ or $r-1$, Eq. (21) can be used to obtain critical point solutions for $X$. Additionally, the remaining variables can be expressed in terms of $X$. When $Z_{c}=0$, it is straightforward to obtain a trivial critical point solution at the origin. For $Z_{c}=r-1$, the system has two sets of nontrivial critical point solutions. By obtaining the critical point solutions using Eqs. (21)-(23), we compute the solution of temperature $(\theta)$ in the physical space (e.g. Eq. (8) of Shen, 2014 for the 5DLM) and compare it with those in the 3D, 5D, and 7D LMs. Figure 1 clearly indicates that a high-dimensional LM with additional modes at higher vertical wavenumbers provides an improved vertical representation of temperature.

Below, we discuss the aggregated nature of negative feedback by comparing the solutions of critical points among the 5D, 7D, and 9D LMs which share the same equation as Eq. (22) that includes a different degree of complexity in $T_{1}$ and $T_{2}$. Specifically, the pairs of $\left(T_{1}, T_{2}\right)$ for the $7 \mathrm{D}$ and $5 \mathrm{D}$ LMs are written as:

$$
\left(T_{1}=\frac{2 X^{2}+b d_{1}}{X^{2}+b d_{1}}, T_{2}=b d_{o} T_{1}\right)
$$

and

$$
\left(T_{1}=1, T_{2}=b d_{o} T_{1}\right),
$$

respectively. In Eqs. (23) and (24) for the 9D and $7 \mathrm{D}$ LMs, respectively, $T_{1}$ is a rational function with a numerator and a denominator that are polynomials with the same degree in $X$. By viewing $T_{1}$ of the 5DLM [Eq. (25)] as a "rational" function with the same numerator and denominator, Eqs. (23)(25) suggest that the degree of the polynomials within higher-dimensional LMs is higher, leading to an increased complexity for $Y_{1}$ in Eq. (22). The results indicate an aggregated feedback. Note that Eq. (23) includes three parameters, $d_{o}, d_{1}$, and $d_{2}$ that appear as coefficients of dissipative terms in association with $Y_{1}, Y_{2}$, and $Y_{3}$ in Eqs. (15), (17), and (19), respectively, but cannot be reduced to Eq. (24) by simply choosing $d_{2}=0$. Similarly, Eq. (24) cannot be reduced to Eq. (25) by plugging $d_{1}=0$ into Eq. (24). This indicates the impact of nonlinear coupled terms between the $Y_{j}$ and $Z_{j}$ modes.

From a perspective of physical processes, the additional Fourier modes that are added to extend the nonlinear feedback loop of the GLM introduce additional dissipative terms. Specifically, within the Rayleigh-Benard convection equations, dissipations are represented by the Laplacian of temperature (e.g. $\nabla^{2} \theta$ in Eq. (2) of Shen, 2014]). After transforming this term using Fourier modes, the coefficients of dissipative terms are proportional to the square of the (vertical or horizontal) wavenumbers. Namely, the higher wavenumber modes have larger coefficients for the dissipative terms. Note that by comparison, the coefficients of the nonlinear terms are proportional to the product of the horizontal and vertical wavenumbers. The above analysis suggests that $X Y_{1}$ within the 9DLM represents the aggregated "dissipative" impact from high wavenumber models and that the aggregated negative feedback produces a stronger effective dissipation since additional high wavenumber modes introduce additional dissipative terms. A simple illustration is provided below.

Assuming that the solution becomes highly nonlinear with a very large $X, T_{1}$ in Eqs. (23)-(25), which approximates 3,2 , and 1 , yields a term $X Y_{1}$ of $3 b Z, 2 b Z$, and $b Z$ for the $9 \mathrm{D}, 7 \mathrm{D}$, and $5 \mathrm{D} \mathrm{LMs}$, respectively. As a result, since $d Z / d \tau$ is proportional to $-X Y_{1}$, a stronger negative feedback with a stronger dissipation appears in higher-dimensional 


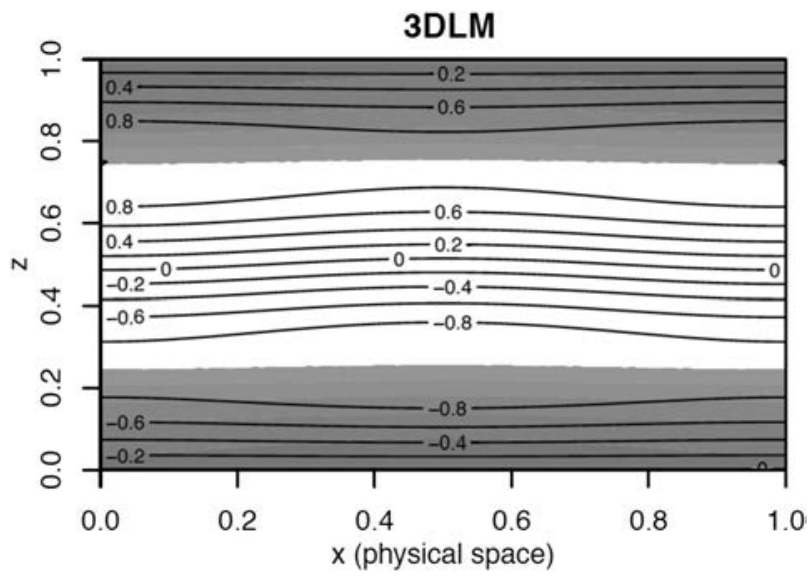

(a)

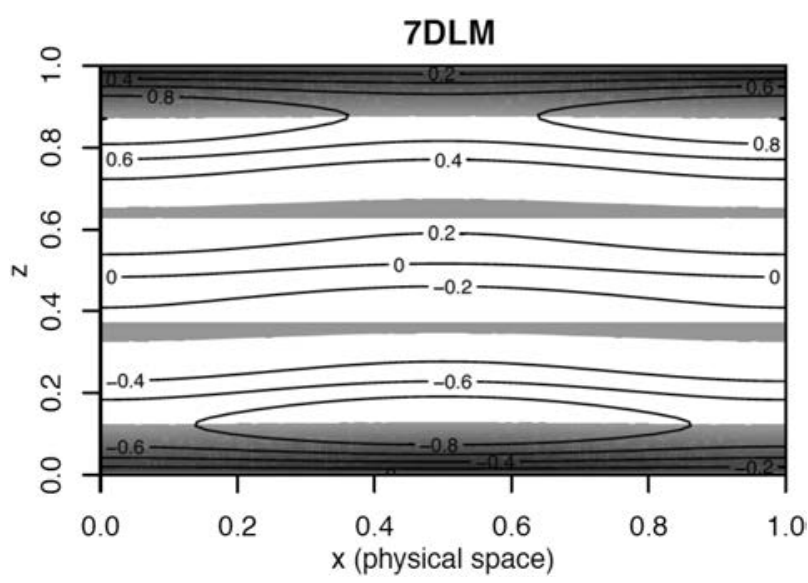

(c)

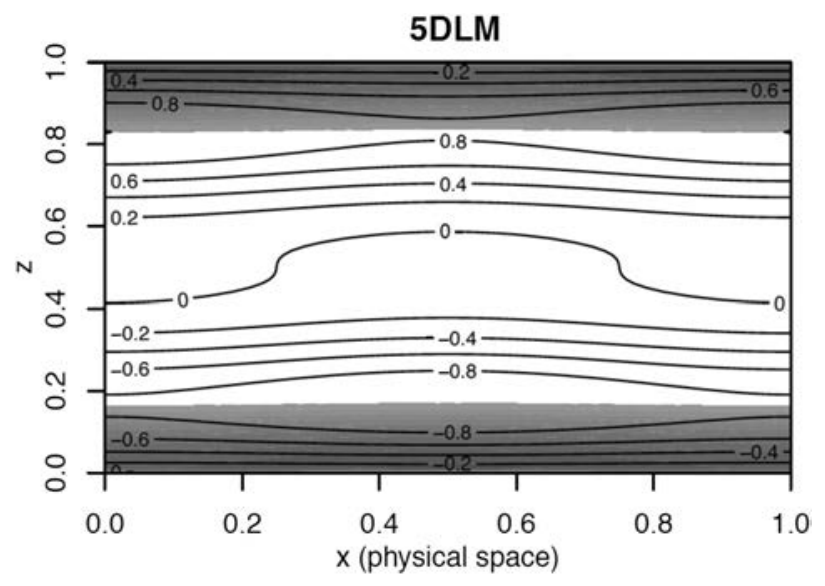

(b)

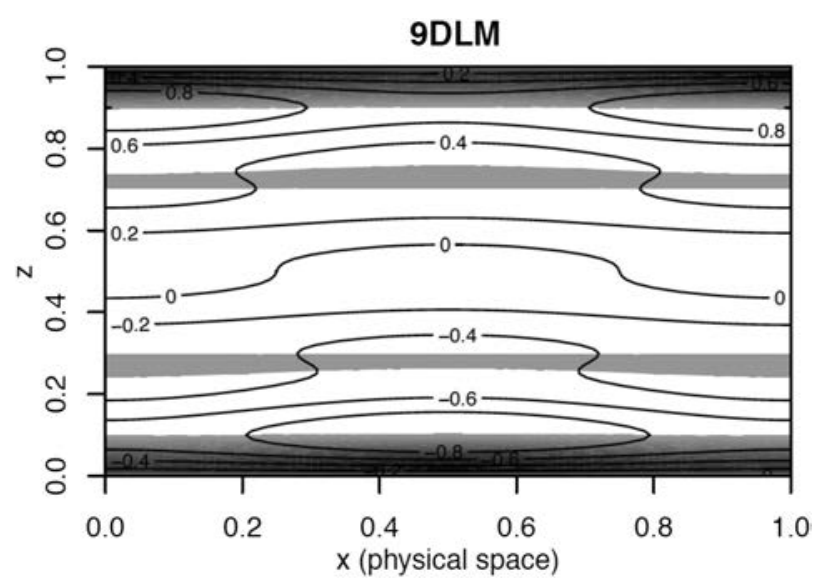

(d)

Fig. 1. Temperature in the physical space $(x, z)$, computed using solutions of the critical points within the (a) 3DLM, (b) 5DLM, (c) 7DLM and (d) 9DLM.

LMs. An interesting feature is that an individual pair of $\left(Y_{j}, Z_{j}\right), j \in[1,3]$ within the 9DLM, seems to contribute a comparable (long-term) negative feedback on the order of $b Z$, although the coefficients of the dissipative terms are proportional to the square of the wavenumbers. Namely, a pair of higher wavenumber modes $\left(Y_{j}, Z_{j}\right)$ contributes a (long-term) negative feedback comparable to lower wavenumber modes (i.e. $Y_{j-1}, Z_{j-1}$ ). Note that the assumption of strong nonlinearity implies that the amplitudes of the solution are not small and, thus, the orbit cannot be close to the origin. The above results may be more applicable near nontrivial critical points. Based on the discussion, we suggest that the aggregated negative feedback associated with successive extension of the nonlinear feedback loop may eventually change the stability of the nontrivial critical point, turning an unstable critical point in a lower-dimensional LM into a stable critical point in a higher-dimensional LM. Below, we expand the discussion.

\subsection{A linear stability analysis within the 9DLM}

By applying the critical point solution as the basic state to linearize the 9DLM [Eqs. (12)-(20)], we perform a linear stability analysis in order to reveal the stability of nontrivial critical points. In Fig. 2, with Prandtl numbers $(\sigma \in[5,50])$ and Rayleigh parameters $(r \in[50,1000])$ in the horizontal and vertical axes, respectively, we plot the contour line of leading eigenvalues with the zero real part in black. The vertical lines in blue and red in Fig. 22 indicate values of $\sigma=10$ and $\sigma=12$, respectively. No intersection between the blue and black lines in Fig. 2 suggests that the real parts of the leading eigenvalues for $\sigma=10$ cannot be zero. In other words, there is no 


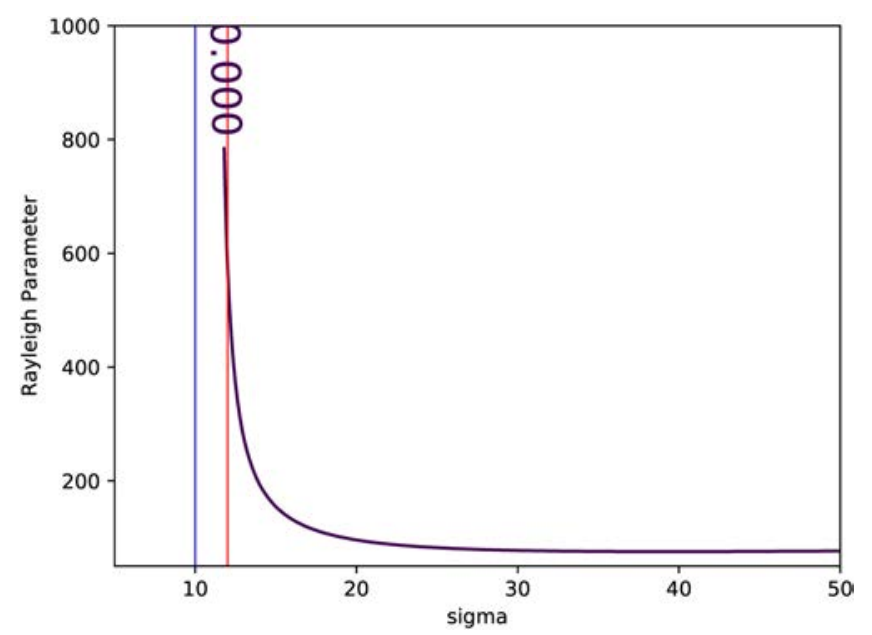

Fig. 2. Stability analysis of the locally linear 9DLM with a $\triangle \sigma$ of 0.1 and a $\triangle r$ of 0.1 . The leading eigenvalue $\operatorname{Re}(\lambda)$ as a function of $\sigma$ and $r$. The black curve indicates a constant contour of $\operatorname{Re}(\lambda)=0$. The blue and red lines indicate $\sigma=10$ and 12 , respectively.

Hopf bifurcation associated with a change of the Rayleigh parameter within the 9DLM with $\sigma=10$. To have unstable nontrivial critical points, $\sigma$ should be close to or larger than 12 , as indicated by the reference line in red. A more detailed analysis is provided in the following paragraphs.

Here, we provide a mathematical analysis to show why the linearized 9DLM with $\sigma=10$ may not have unstable, nontrivial critical points. The right-hand side of Eq. (14), which describes the time evolution of the primary mode $Z$, includes two nonlinear terms $X Y, X Y_{1}$, and one linear dissipative term, $b Z$. The nonlinear term $X Y_{1}$, which involves a secondary mode $\left(Y_{1}\right)$, can be approximated with the critical point solutions of primary modes, as discussed in Eq. (22) . Furthermore, a simplified representation for $X Y_{1}$ may be obtained by analyzing the normalized differences between $X Y_{1}$ and $3 b Z$ over the range of Rayleigh parameters in Fig. 3, as follows:

$$
X Y_{1} \sim 3 b Z,
$$

for a larger $r$. The above equation indicates that the aggregated effect represented by the nonlinear $X Y_{1}$ is comparable to a dissipative term, $3 b Z$, with a coefficient of 3b. Plugging Eq. (26) into Eq. (14), we obtain the following approximation:

$$
\frac{d Z}{d \tau}=X Y-3 b Z-b Z=X Y-4 b Z
$$

Now, the effect of $X Y_{1}$ is emulated (or "parameterized") by the term $3 b Z$ (or by the primary mode $Z$ ).
Thus, Eq. (27) is an approximation of Eq. (14) and does not explicitly include any high wavenumber modes. Compared to $d Z / d \tau$ of the 3DLM [i.e. no $X Y_{1}$ in Eq. (14)], Eq. (27) includes a linear dissipative term with a larger coefficient. Equations (12) and (13) and Eq. (27) form a new system with additional feedback from high wavenumber modes for describing the time evolution of primary modes $(X, Y$, and $Z$ ). Applying the same approach used in Lorenz, 1963], a linearization of the new system with respect to its nontrivial critical point leads to the following characteristic equation:

$$
\begin{aligned}
\lambda^{3}+ & (\sigma+4 b+1) \lambda^{2}+4 b(\sigma+r) \lambda \\
+ & 8 \sigma b(r-1)=0
\end{aligned}
$$

here, $\lambda$ is the eigenvalue associated with linear solutions in the form of $\exp (\lambda \tau)$. Note that Eq. (28) can be obtained by replacing $b$ by $4 b$ in Eq. (33) of Lorenz, 1963]. A necessary condition for the Hopf bifurcation can be obtained when eigenvalues $(\lambda)$ include pure imaginary numbers (i.e. $\operatorname{Re}(\lambda)=0$ ), yielding:

$$
\sigma>4 b+1
$$

The above leads to $\sigma>11.67$ when $b=8 / 3$. Thus, to have unstable nontrivial critical points, $\sigma$ must be larger than 11.67. The analysis with the approximate relation in Eq. (26) is consistent with the above linear stability analysis shown in Fig. 2

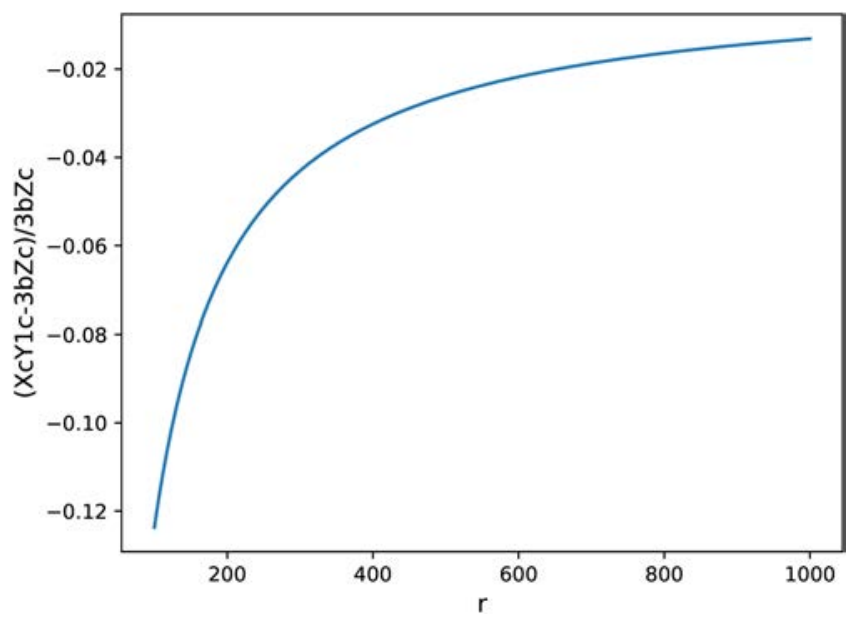

Fig. 3. An estimate of the feedback term, $X Y_{1}$, using the nontrivial critical point solution. The estimate shows the normalized differences between the $X_{c} Y_{1 c}$ and $3 b Z_{c}$. Within a wide range of Rayleigh parameters $(r)$, the normalized differences are within 10\%, suggesting the relationship $X Y_{1} \sim 3 b Z$ that is used in Eq. (27). 
Note that: (i) as a result of the simplified approach by approximating $X Y_{1}$ using steady-state critical point solutions, the above results may better represent long-term feedback of dissipative processes at small scales; (ii) time-varying feedback during an initial (short) period of time may be complicated in association with multiple frequencies produced by extensions of the NFL; and (iii) since additional nonlinear terms in association with the NFL are introduced, the location of nontrivial critical points are different in various LMs (e.g. $X_{c}=$ $\sqrt{b(r-1)}$ and $X_{c} \sim \sqrt{2 b(r-1)}$ for the 3DLM and 5DLM [e.g. Eq. (20b) of [Shen, 2014]], respectively). The impact of such changes is the subject of a future study.

\subsection{An analysis of Lyapunov exponents from 10000 ensemble runs}

A system is said to be chaotic when it has a positive LE and bounded solutions. To determine under which condition chaotic solutions may appear in our LMs, Lyapunov exponents (LEs) were computed over a range of Rayleigh parameters. For a given $r$ in the control run, referred to as the CNTL run, 10000 different initial conditions (ICs) were created as Gaussian white noise with a mean of zero and a standard deviation of one [Press et al., 1992]. Each of the 10000 ICs within the control run generates one Lyapunov exponent. The average of the 10000 LEs is computed and is referred to as an ensemble Lyapunov exponent (eLE). A transition from a negative eLE to a positive eLE was used to detect the onset of chaos within the 5D and 7D LMs Shen, 2014, 2016]. The critical values of the Rayleigh parameter $\left(r_{c}\right)$ are 42.9 and 116.9 for the 5D and 7D LMs, respectively. While a detailed analysis of eLEs for the 9DLM is presented later, a summary for major results presented in Fig. 4 is provided here: (1) the $r_{c}$ of the 9DLM is approximately 679.8, as shown with open blue circles; (2) an effective upper bound $\left(r_{d}=1058\right)$ for Rayleigh parameters for chaotic solutions is shown with green or pink lines; (3) for $r \in\left[r_{c}, r_{d}\right]=[679.8,1058]$, some "windows" with nonpositive eLEs appear within a small interval of Rayleigh parameters; and (4) when $r$ becomes larger, periodic solutions (e.g. $r=1100$ )

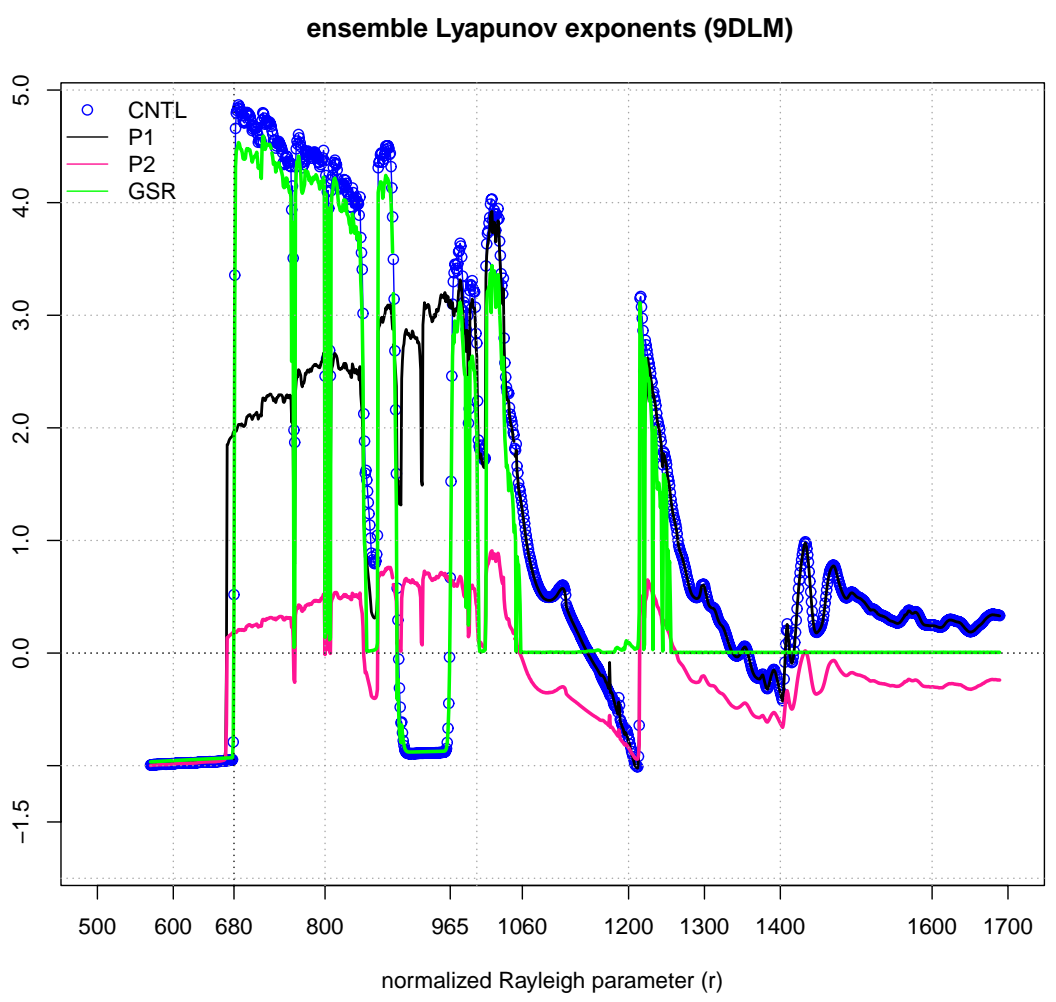

Fig. 4. The largest ensemble-averaged Lyapunov exponents (eLEs) as a function of the Rayleigh parameter, $r$, using the 9DLM. The figure provides results for $\triangle r=1$. The appearance of chaotic solutions is indicated by positive eLEs. Note that (1) the critical value of $r$ for the onset of chaos in the 9DLM is between $r=679$ and $r=680$; (2) zero values of eLEs associated with "periodic" solutions are better shown using the GSR method in green. 
or limit cycles (e.g. $r=1600$ ) with nearly zero eLEs in green may coexist with steady-state solutions.

To assure the robustness of our results, we perform two parallel runs in which the 10000 ICs of the control run are multiplied by 100 or 500, here, referred to as the P1 and P2 runs, respectively. Parallel runs allow ICs to be distributed over a larger space. Within the parallel runs, some ICs become closer to the nontrivial critical points. For calculation of the eLE, the orbit separation (OS) method Sprott, 2003] is used for the CNTL, P1, and P2 runs, shown in blue circles, and black and pink lines, respectively. For comparison, the Gram-Schmidt reorthonormalization (GSR) procedure Wolf et al., 1985) is used in an additional run using the same ICs as those in the CNTL run, referred to as the GSR run that is shown with a green line. For control and parallel runs using the OS method, the $r_{c}$ values are comparable. The range of Rayleigh parameters for chaotic solutions are also comparable. However, within the regime of chaotic solutions, the parallel runs display much smaller eLEs as compared to the control run. The dependence of the magnitude of an eLE on the spatial extent of ICs in ensemble members, as well as the comparison of results using the OS and GSR methods, is discussed in the following paragraphs.

In the histogram analysis of 10000 LEs for a given $r$, we choose a bin size of 0.1 (i.e. $\Delta L E=0.1$ ) for the horizontal axis. In Fig. 5(a), a histogram with $r=680$ shows two clusters: one displays negative values of $[-0.9,-1.0]$ and the other displays positive values of $[4.8,5.0]$. The result, in

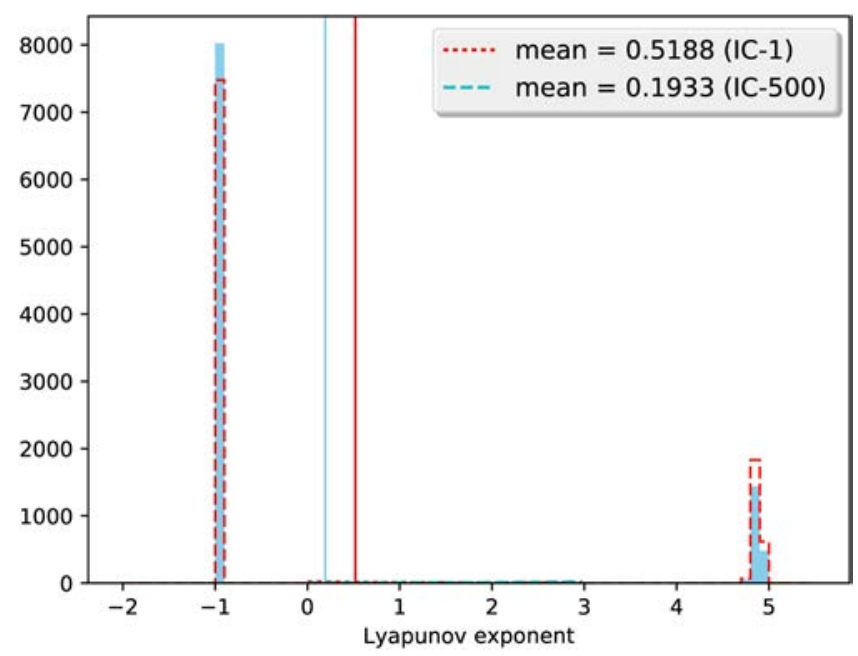

(a) fact, indicates the coexistence of two types of solutions: steady-state solutions with a negative LE and chaotic solutions with a positive LE. Since the 10000 ensemble runs share the same model configurations but have different ICs, the appearance of either a steady-state or chaotic solution is solely determined by an initial condition. Within the control run, the first and second clusters have approximately 7470 and 2400 members, respectively, and, thus, the probability for the appearance of chaotic orbits is roughly $24 \%$. In comparison, the P2 run has 8000 and 1900 members for the first and second clusters, respectively. The probability for the occurrence of chaotic orbits is roughly $19 \%$, which is smaller than that in the control run. Since the P2 run includes ICs that were distributed over a larger space, some ICs are closer to the stable nontrivial critical points and produce larger number of steady-state solutions. Thus, the P2 run has a lower percentage of chaotic orbits and a smaller eLE.

An additional comparison of the control runs with $r=680$ and $r=720$, as shown in Figs. 5(a) and 5(b), indicates that a higher percentage of chaotic solutions appear at a larger $r$. For the control run with $r=720$, most of the ensemble runs produce chaotic orbits. However, when the ICs are distributed over a larger space [e.g. the P2 run in Fig. 5(b)], a significant number of steady-state orbits are simulated. Since the two types of solutions produce very different LEs and since an eLE is computed as an ensemble average, the eLE displays a dependence on the ratio of the number of chaotic and steady-steady solutions. Alternatively

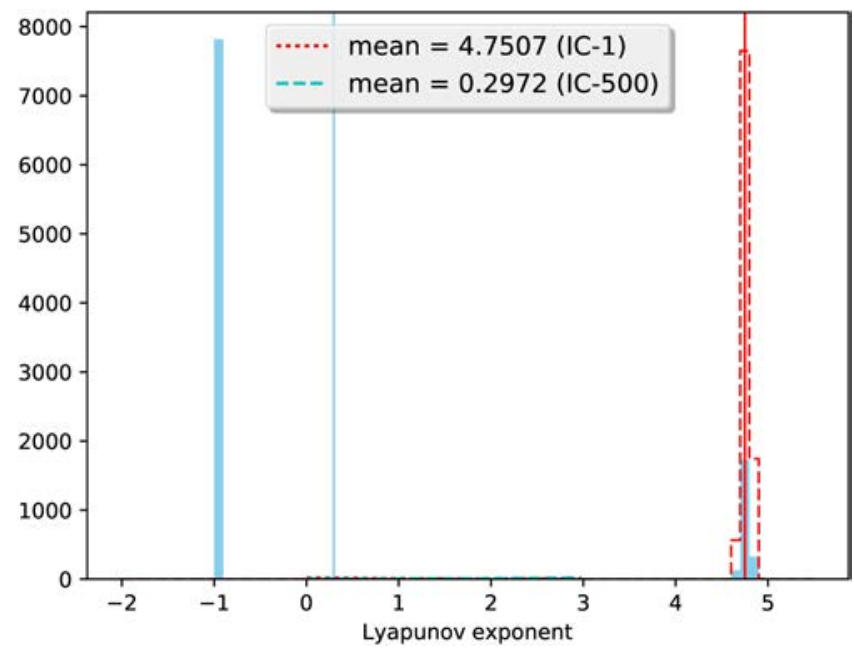

(b)

Fig. 5. Histogram of 10000 Lyapunov exponents from runs with (a) $r=680$ and (b) $r=720$. 
speaking, the dependence of the eLE's magnitude on the spatial extent of ICs may indicate the coexistence of two types of solutions. Among the CNTL, P1 and P2 runs, a smaller eLE is obtained when 10000 ICs are distributed over a larger phase space and, thus, the P2 run produces the smallest eLE within the regime of chaotic solutions, as shown in Fig. 4.

In the above, we discussed how the eLE analysis helps determine the critical value for the onset of chaos. Here, we continue to determine an upper limit for the Rayleigh parameter for chaotic solutions. As shown for the 3DLM, doing so is a challenging task. For example, the 3DLM is known to produce chaotic solutions when $r>24.74$ and limit cycle solutions when $r>313$ (as suggested bv [Sparrow, 1982]). However, for $r$ in between these two values (e.g. $r \in[166,214.36])$ either limit cycle or chaotic solutions may appear within the 3DLM (e.g. Fig. 8.4 of [Drazin, 1992]). Additionally, for $r=160$, the 3DLM produces a "periodic" but not a simple harmonic solution (e.g. Fig. 1.20 of [Hilborn, 1994]). As discussed below, this type of solution also appears within the 9DLM with a much larger $r$. As a result, the following discussions determine an effective upper bound $\left(r_{d}\right)$ of Rayleigh parameters for chaotic solutions and an effective lower bound $\left(r_{e}\right)$ of Rayleigh parameters for limit cycle solutions. For $r \in\left[r_{d}, r_{e}\right]$, we show some interesting solutions for revealing challenges associated with the related analyses.

In Fig. 4, the P2 run shows negative eLEs when $r \in[1057,1148]$, while both the CNTL and P1 runs still display positive eLEs. The results may indicate that most ensemble members within the P2 run produce steady-state solutions with negative LEs, while a significant number of ensemble members within the CNTL and P1 runs produce orbits with "positive LEs". However, the difference may provide additional information. By comparison, the GSR run, using the GSR method, produces nearlyzero eLEs between $r=1058$ and $r=1215$, which is also different from all runs using the OS method. By analyzing the results, as shown in Fig. 7 with $r=1100$, both "periodic" and steady-state solutions appear. Due to the same ICs, a comparison between the CNTL and GSR runs suggests that LEs for "periodic solutions" may be better computed using the GSR method. Therefore, the GSR run is used to determine an effective upper bound $\left(r_{d}=1058\right)$ of Rayleigh parameters for chaotic solutions, which is comparable to the one estimated using the P2 run.

For large Rayleigh parameters, $r \in[1058,1700]$ and $r \notin[1215,1250]$, eLEs from the GSR method display nearly-zero values, as indicated with the green line in Fig. 4. In comparison, within the CNTL, P1, and P2 runs, some eLEs are negative while others have positive values that, however, are smaller than those for chaotic solutions. Our numerical results with $r>r_{d}$ indicate that the 9DLM produces the coexistence of steady-state solutions with various types of "periodic" solutions, including limit cycle solutions with an isolated nature. In Sec. 3.4, we present some of these solutions. For the coexistence of various "periodic" solutions, it may be challenging to compute corresponding LEs using the OS method and, thus, the eLE. Although the GSR method may produce reasonable values for "periodic" solutions, this method cannot determine if the "periodic" solutions are LC type solutions with an isolated nature. As a result of complexity, although we suggest that limit cycle solutions may appear $r>r_{e}=1550$, based on our preliminary analysis, we want to emphasize that LC solutions may also appear in $r \in\left[r_{d}, r_{e}\right]=[1058,1550]$. We provide additional details below.

\subsection{Numerical solutions for the coexistence of two types of orbits}

The above discussions suggest the dependence of chaotic or nonchaotic solutions on initial conditions. Here, after analyzing some simulations, we present two runs to illustrate the dependence. In Fig. 6 where $r=680, \sigma=10$, and $b=8 / 3$, panels (a) and (b) display solutions using two different initial conditions, $\left(X, Y, Z, Y_{1}, Z_{1}, Y_{2}, Z_{2}, Y_{3}, Z_{3}\right)=(0,1,0$, $0,0,0,0,0,0)$ and $(100,100,100,100,100,100,100$, 100,100), respectively. The former produces a steady-state solution, approaching one of the stable nontrivial critical points. The latter leads to an irregularly oscillatory solution. Since a linear stability analysis suggests one saddle point at the origin and two stable critical points, irregular oscillations seem related to the saddle point at the origin. Note that the "butterfly pattern" of the 9DLM, whose width is thinner, indeed looks (slightly) different from the one obtained using the 3DLM.

In the following, we present numerical experiments for $r>r_{d}=1058$. Figure 7 displays the coexistence of a "periodic" and steady-state solution 


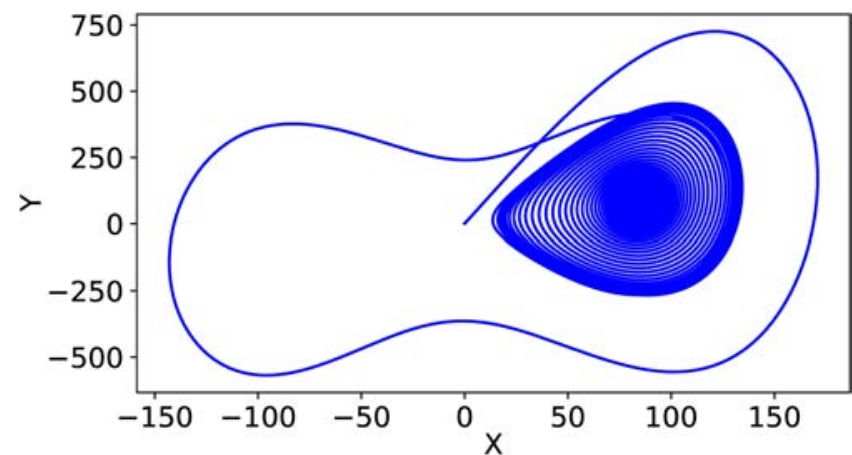

(a)

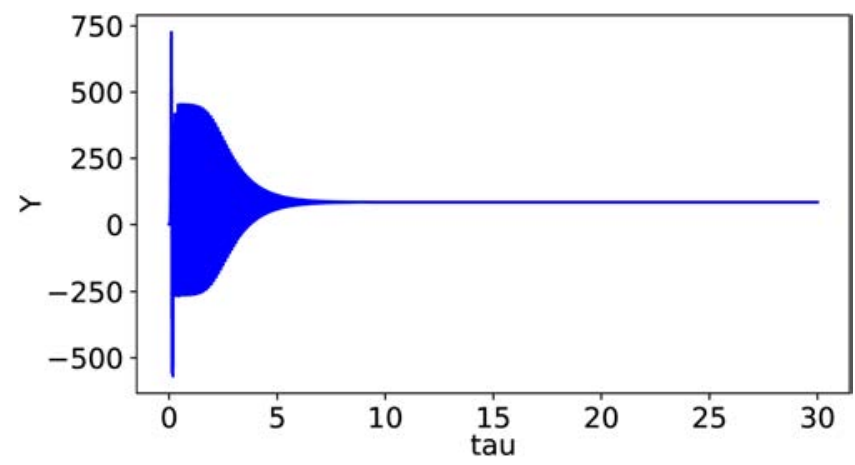

(c)

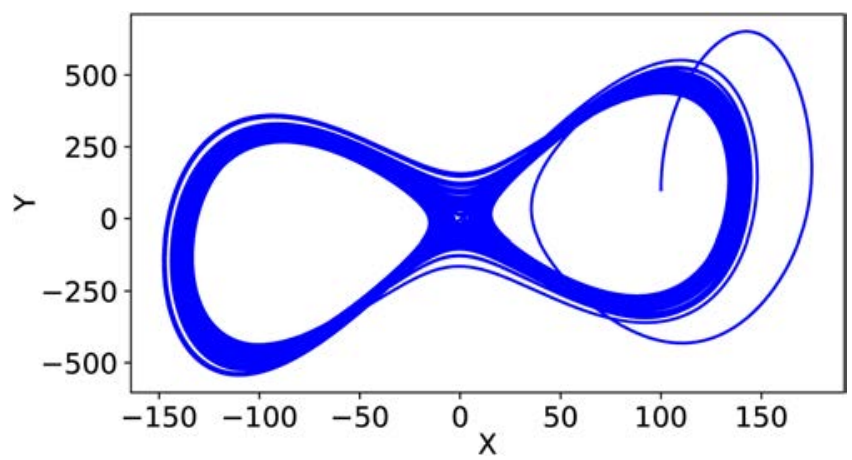

(b)

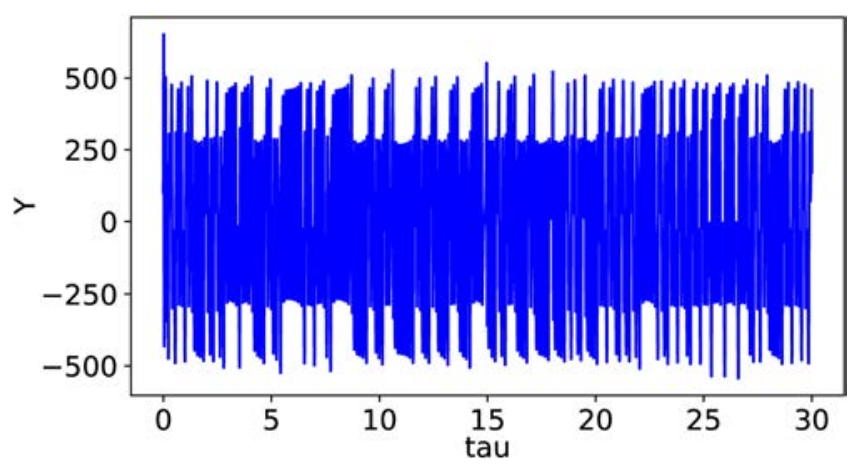

(d)

Fig. 6. Coexistence of nonchaotic (left panels) and chaotic (right panels) solutions. The two runs use the same parameters (including $r=680)$ but different initial conditions, which are $\left(X, Y, Z, Y_{1}, Z_{1}, Y_{2}, Z_{2}, Y_{3}, Z_{3}\right)=(0,1,0,0,0,0,0,0,0)$ and $(100,100,100,100,100,100,100,100,100)$, respectively.

using the same model configurations (e.g. $r=1100$ ) but different ICs. In Fig. 8 where $r=1120$, in addition to the steady-state solutions, the 9DLM produces two types of "periodic" solutions: one displays a large cycle and the other appears to include both large and small cycles. The latter is similar to the solution of the 3DLM with $r=160$. One common feature of the "periodic" orbits in Figs. 7 and 8 is that they do not move close to the origin. Since the Limit cycle (LC) is an isolated closed orbit, it represents one special kind of periodic orbit. However, a periodic orbit does not necessarily possess the isolated nature of a LC. For each of the above periodic orbits in Figs. 7 and 8 additional

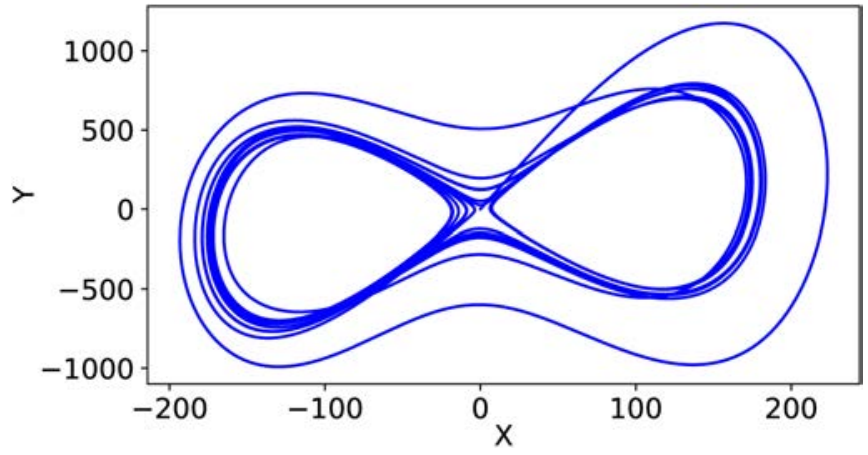

(a)

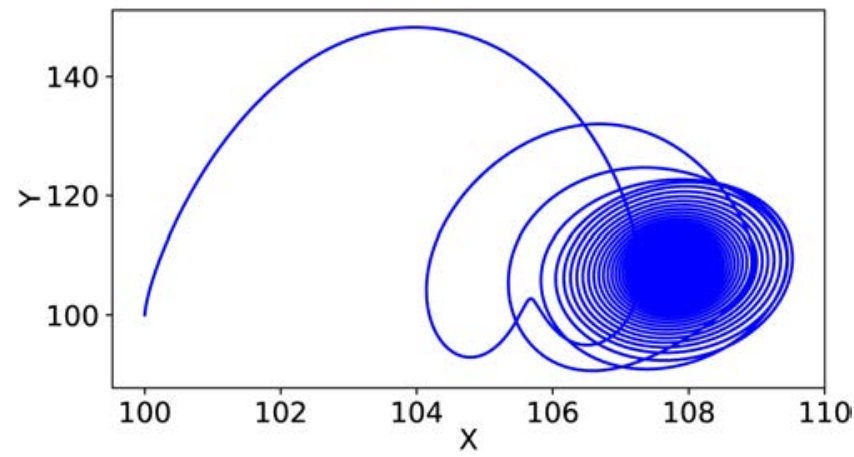

(b)

Fig. 7. Coexistence of "periodic" (left panels) and steady-state (right panels) solutions. The two runs use the same parameters (including $r=1100)$ but different initial conditions, which are $\left(X, Y, Z, Y_{1}, Z_{1}, Y_{2}, Z_{2}, Y_{3}, Z_{3}\right)=(0,1,0,0,0,0,0,0,0)$ and $(100,100,1100,100,550,100,330,100,260)$, respectively. 


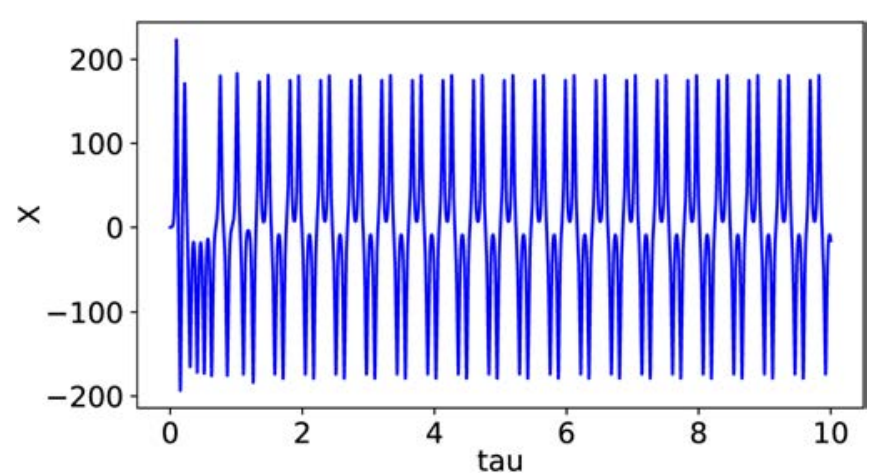

(c)

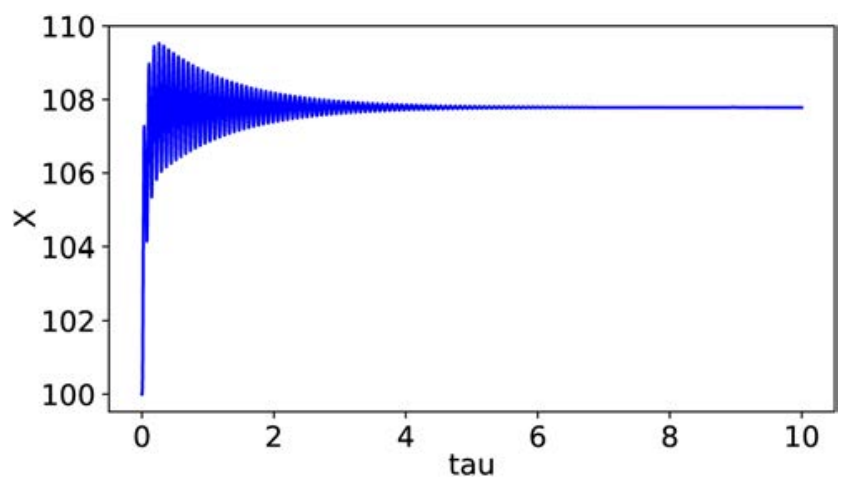

(d)

Fig. 7. (Continued)

parallel runs with 20 ensemble members suggest that the aforementioned periodic orbits are not isolated, and, thus, are not LC orbits (not shown).

As a result of the complexity of the system for large Rayleigh parameters, here, we simply illustrate one case for the LC orbit as a comparison with the "periodic" orbits in Figs. 7 and 8. LC solutions appear at large Rayleigh parameters within the 3DLMs, 5DLMs, and 7DLMs [Shen \& Faghih-Naini, 2017]. As shown in Fig. 9 for large Rayleigh parameters within the 9DLM, LC and steady-state solutions coexist in runs with the same $r=1600$ but with different initial conditions, $\left(X, Y, Z, Y_{1}, Z_{1}, Y_{2}, Z_{2}, Y_{3}\right.$, $\left.Z_{3}\right)=(0,1,0,0,0,0,0,0,0)$ and $(100,100,1600,100$, $800,100,530,100,400)$, respectively. To illustrate

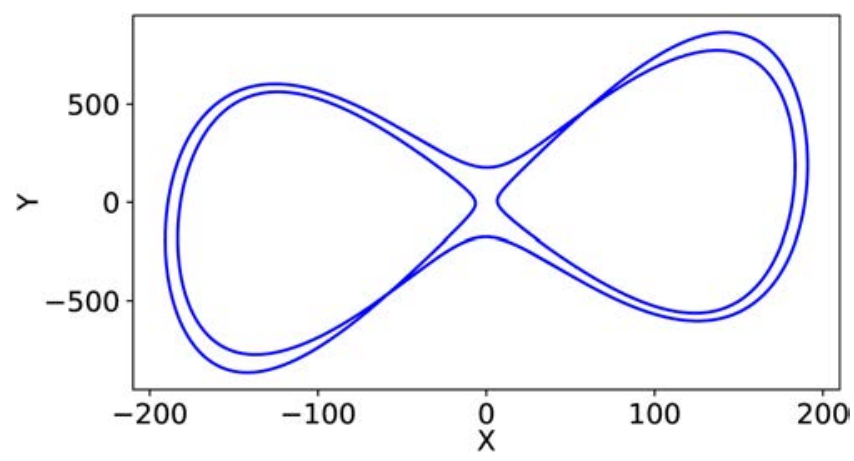

(b)

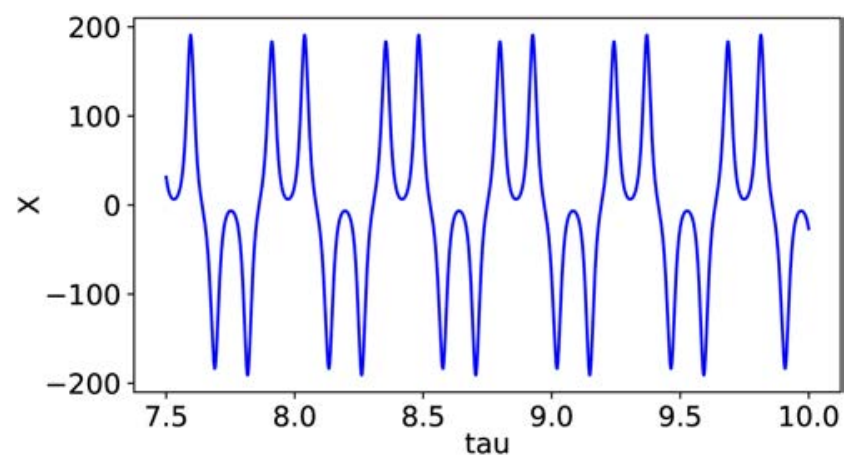

(d)

Fig. 8. Two types of "periodic" solutions. The two runs use the same parameters (including $r=1120$ ) but different initial conditions, which are $\left(X, Y, Z, Y_{1}, Z_{1}, Y_{2}, Z_{2}, Y_{3}, Z_{3}\right)=(0,1,0,0,0,0,0,0,0)$ and $(40,557,1123,94,156,714,386,507,140)$, respectively. 


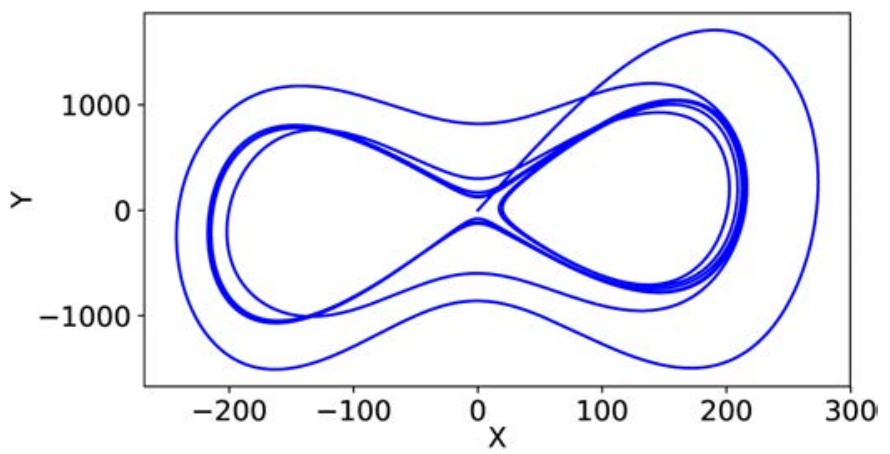

(a)

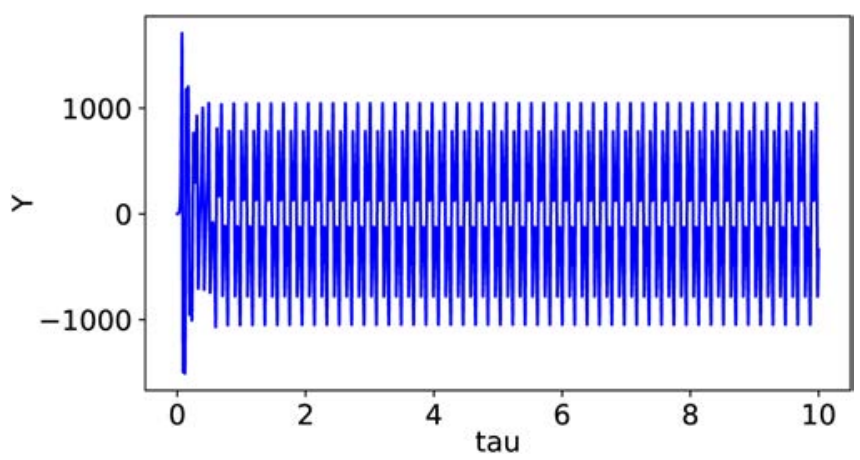

(c)

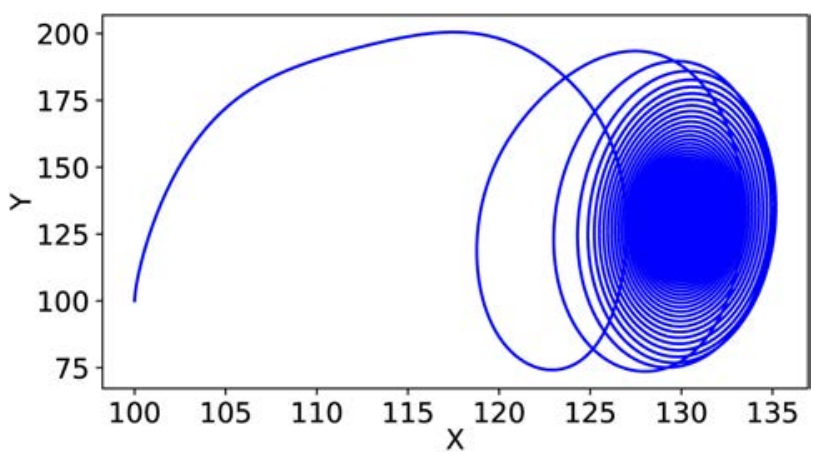

(b)

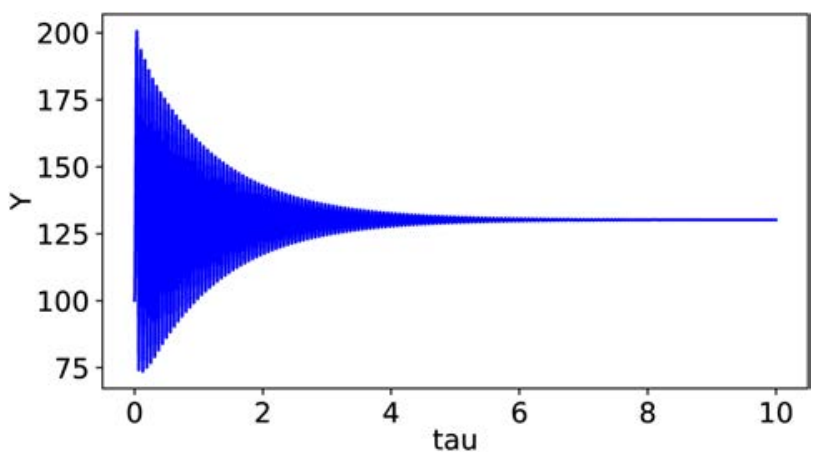

(d)

Fig. 9. Coexistence of limit cycle (left panels) and steady-state (right panels) solutions. The two runs use the same parameters (including $r=1600)$ but different initial conditions, which are $\left(X, Y, Z, Y_{1}, Z_{1}, Y_{2}, Z_{2}, Y_{3}, Z_{3}\right)=(0,1,0,0,0,0,0,0,0)$ and $(100,100,1600,100,800,100,530,100,400)$, respectively.

the interesting "isolated" feature of the LC solution, as well as its coexistence with steady-state solutions, Fig. 10provides solutions from 2048 ensemble runs that only differ in initial conditions. Generated

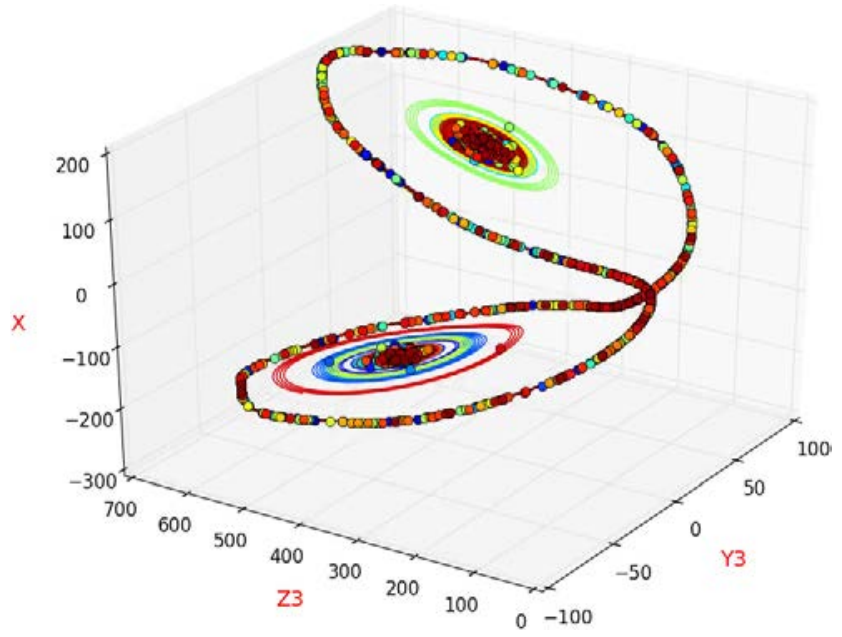

Fig. 10. Time evolution of 2048 orbits in the $X-Y_{3}-Z_{3}$ phase space using the 9DLM, showing two spiral sinks and a limit cycle/torus solution. The animation is available at https://goo.gl/sMhoUb. as Gaussian random variables, the initial conditions are distributed over a hypersphere as follows:

$$
\begin{aligned}
W_{i j} & =R \frac{V_{i j}}{\text { norm }_{j}}, \quad i \in[1,9] ; j \in[1,2048], \\
\operatorname{norm}_{j} & =\sqrt{\sum_{i=1}^{9} V_{i j}^{2}}, \quad j \in[1,2048],
\end{aligned}
$$

which yield:

$$
\sum_{i=1}^{9} W_{i j}^{2}=R^{2}, \quad j \in[1,2048] .
$$

Here, $i \in[1,9]$ indicates each of the nine variables within the phase space, while $j \in[1,2048]$ is within the ensemble space. Therefore, $W_{1 j}, W_{2 j}, W_{3 j}, \ldots$, $W_{8 j}, W_{9 j}$ represent a set of ICs for $X, Y, Z, \ldots$, $Y_{3}, Z_{3}$, respectively. $R$, the "radius" of the hypersphere, represents the spatial extent of the initial conditions within the phase space. We choose $R$ to be proportional to the Rayleigh parameter. The total simulation time is $T=3.5$. The still image in 
Fig. 10 is located near the end of the simulation. Some of the 2048 different orbits spiral inward to one of two stable nontrivial points, while others are attracted to an LC with a large closed orbit. Along the LC orbit, each color dot corresponds to one initial condition. The isolated nature of the LC can best be shown with an animation, available at https://goo.gl/sMhoUb. Transient orbits are only kept for the last 0.25 time units (i.e. for the time interval of $[\max (0, \tau-0.25), \tau]$ at a given time $\tau$ ). The zoom-in of the domain begins at $\tau=0.25$ and ends at $\tau=0.45$, leading to a smooth domain change from $\left(X, Y_{3}, Z_{3}\right)=(-1300,1200) \times$ $(-1100,1100) \times(-1000,1700)$ to $(-300,200) \times$ $(-100,100) \times(0,700)$.

As mentioned, simplified versions of the Lorenz model can be used to illustrate the fundamental dynamics of the model. Table 1 lists three types of simplified models. A local analysis near the origin shows that the geometric model seems suitable for understanding behaviors of solutions near the origin. As discussed in [Shen, 2018], the limiting equations represent a special version of the nondissipative Lorenz model with $r=0$. A closed-form solution to the limiting equations (e.g. Eq. (18) of Shen, 2018]) reveals the role of the nonlinear terms in producing oscillatory solutions. Thus, the limiting equations may be better for representing solutions near a nontrivial critical point instead of at the origin which is a saddle point in the original Lorenz model. Thus, the degree of how close an orbit returns back to the origin may help reveal the major differences between the chaotic and LC orbits. Such is illustrated by comparing the chaotic and LC solutions of the 9DLM. From Figs. 6(b) and 9(a), one of the major differences between the chaotic and LC orbits is that the former can return very close to the origin, while the latter cannot. The comparison of the two types of solutions qualitatively displays the validity of the geometric model and limiting equations for revealing the divergent and oscillatory characteristics of chaotic and LC orbits, respectively. While the geometric model emphasizes the collective impact of linear heating and dissipation, the limiting equations and the nondissipative Lorenz model focus on the collective impact of the nonlinearity and heating. A comparison of the results from the three simplified models suggests a conceptual model for the appearance of the LC orbit in the original Lorenz model: (1) The oscillatory nature of the LC is enabled by the collective impact of strong heating and nonlinearity; (2) For the "isolated nature" of the LC, a relatively small dissipation is required; and (3) As a result, for the returning orbit where nonlinearity and heating may dominate, the relative small dissipation may not be strong enough to "attract" the orbit toward the origin.

\section{Conclusions}

In this study, we presented a generalized Lorenz model (GLM) with $M$ modes, where $M$ is an odd number that is greater than three. The GLM is derived through successive extension of the nonlinear feedback loop (NFL), a generalized approach based on the work of Shen [2014] for the 5DLM and Shen 2016] for the 7DLM. We discussed major characteristics of the GLM with nine modes (i.e. the 9DLM). By comparing solutions of the critical points within the GLM with various modes, we found (1) that aggregated negative feedback is enabled by successive extension of the NFL using additional high wavenumber modes and (2) that the 9DLM represents a minimal set of ODEs for a Lorenz-type model that possesses stable, nontrivial critical points for $r>1$ as well as $\sigma=10$ and $b=8 / 3$. Within the 9DLM, we discussed two unique features of the 9DLM: (1) the coexistence of chaotic and nonchaotic orbits with moderate Rayleigh parameters (e.g. $679.8 \leq r<r_{d}=1058$ ) and (2) the coexistence of limit cycle orbits and spiral sinks with large Rayleigh parameters (e.g. $\left.r \geq r_{e}=1550\right)$. The first feature can be found in lower-dimensional LMs but appears over a very small range of $r$ (i.e. $24.06<r<24.74$ within the 3DLM). The second feature has never been discussed in the literature. For $r \in\left[r_{d}, r_{e}\right]$, steady-state solutions, periodic solutions, limit cycle solutions, or two or three types of solutions may appear. A detailed analysis for refining the values of $r_{d}$ and $r_{e}$ is the subject of a future study.

The coexistence of two types of solutions (e.g. chaotic and steady-state solutions) was illustrated using a histogram analysis of 10000 Lyapunov exponents (LEs) for a given $r$, displaying two clusters: one with negative LEs for steady-state solutions and the other with positive LEs for chaotic solutions. Since the ensemble LE (eLE) is an average of 10000 LEs, it depends on the number of ensemble members in each of the two clusters. An eLE decreases when the number of steady-state orbits increases. 
Since the spatial extent of 10000 ICs may determine the ratio of steady-state and chaotic orbits, it could determine the magnitude of the eLE, suggesting the dependence of the eLE on the spatial extent of the 10000 ICs. Such a dependence is an indicator for the coexistence of two types of solutions. Although the eLE of the 9DLM shows dependence on the spatial extent of the initial conditions, the control and parallel runs consistently suggest a critical value for the Rayleigh parameter $\left(r_{c}=679.8\right)$ for the onset of chaos. The critical value, $r_{c}$, for the 9DLM is larger as compared to the $r_{c}=24.74$ for the 3DLM, the $r_{c}=42.9$ for the 5DLM, and the $r_{c}=116.9$ for the 7DLM. Since a higher-dimensional model has a larger $r_{c}$, this feature indicates aggregated negative feedback.

The results obtained from the GLM with various modes confirm the hypothesis that the proper selection of additional Fourier modes for successive extension of the NFL within the high-dimensional LM can provide aggregated negative feedback for stabilizing solutions. The findings suggest that an improved degree of nonlinearity within a real-world, high-resolution model may stabilize solutions, leading to improved predictability. However, a comparison between the GLM (e.g. $M=5$ or $M=7$ ) and the $6 \mathrm{DLM}$ (or 8DLM) indicates that heating effects associated with excessive precipitation in the highresolution model may destabilize solutions, thereby increasing the sensitivity of simulations to small perturbations. It is equally important to understand the performance of physics parameterizations (e.g. heating) and their interactions with model dynamics (i.e. explicit terms) in high-resolution simulations.

The role of the extended NFL in producing recurrent solutions with multiple incommensurate frequencies was revealed using the nondissipative Lorenz models (NLMs) in this study, as well as in [Shen, 2018] and Faghih-Naini \& Shen, 2018]. By transforming the Lorenz system into the Euler rotational equation, the recurrence can be also shown by analyzing the nonlinear terms of conservative force (torque) that represents the inertial part for free rotation Pelino et al. . 2014: Qi. 2017: Qi \& Liang, 2016]. Governing equations for the locally linear 3D-, 5D-, 7D-, and 9D-NLMs are identical to those for systems with one, two, three, and four masses and springs, respectively, yielding one, two, three, and four (incommensurate) frequencies, respectively. The appearance of multiple frequencies seems to complicate solutions, in particular, near the saddle point at the origin. Mathematically, the corresponding components of the linear solutions have zero growth rates because the real parts of the eigenvalues are zero for nondissipative systems. By comparison, when dissipative terms are considered in high-dimensional LMs, strong dissipations associated with high wavenumbers appear. The dissipations can produce negative feedback to change the stability of nontrivial critical points and lead to some decaying components with large negative growth rates (or Lyapunov exponents). High-frequency modes that appear associated with high wavenumber modes could quickly dissipate. Therefore, while the extension of the NFL may increase the complexity of solutions by introducing additional frequencies, the inclusion of additional dissipations at high wavenumbers may reduce the complexity of solutions by dissipating highfrequency components.

As outlined above, high-dimensional LMs possess various types of solutions (e.g. chaotic and limit cycle orbits) over a range of Rayleigh parameters. We hypothesize that a more complicated modeling system that allows the coexistence of various types of chaotic and nonchaotic processes may better represent the nature of weather.

\section{Acknowledgments}

We thank anonymous reviewers for valuable comments, Ms. T. Reyes, Ms. S. Faghih-Naini, and Mr. J. Cui for their help in verifying derivations and producing animations. We are grateful for support from the College of Science at San Diego State University.

\section{References}

Anthes, R. [2011] "Turning the tables on chaos: Is the atmosphere more predictable than we assume?" UCAR Magazine, spring/summer, available at: https://news. ucar.edu/4505/turning-tables-chaos-atmosphere-morepredictable-we-assume (last access: 26 November 2018).

Bender, C. M. \& Orszag, S. A. [1978] Advanced Mathematical Methods for Scientists and Engineers (McGraw-Hill, NY), 593 pp.

Blender, R. \& Lucarini, V. [2013] "Nambu representation of an extended Lorenz model with viscous heating," Physica D 243, 86-91.

Curry, J. H. [1978] "Generalized Lorenz systems," Commun. Math. Phys. 60, 193-204. 
Curry, J. H., Herring, J. R., Loncaric, J. \& Orszag, S. A. [1984] "Order and disorder in two- and three-dimensional Benard convection," J. Fluid Mech. 147, 1-38.

Faghih-Naini, S. \& Shen, B.-W. [2018] "Quasi-periodic in the five-dimensional non-dissipative Lorenz model: The role of the extended nonlinear feedback loop," Int. J. Bifurcation and Chaos 28, 1850072-1-20.

Fay, T. H. \& Graham, S. D. [2003] "Coupled spring equations," Int. J. Math. Educ. Sci. Technol. 34, 65-79.

Felicio, C. C. \& Rech, P. C. [2018] "On the dynamics of five- and six-dimensional Lorenz models," J. Phys. Commun. 2, 025028.

Floratos, E. [2012] "Matrix quantization of turbulence," Int. J. Bifurcation and Chaos 22, 1250213-1-8.

Gleick, J. [1987] Chaos: Making a New Science (Penguin, NY), 360 pp.

Guckenheimer, J. \& Williams, R. F. [1979] "Structural stability of Lorenz attractors," Publ. Math. IHES 50, 59.

Hermiz, K. B., Guzdar, P. N. \& Finn, J. M. [1995] "Improved low-order model for shear flow driven by Rayleigh-Benard convection," Phys. Rev. E 51, $325-331$.

Hirsch, M., Smale, S. \& Devaney, R. L. [2013] Differential Equations, Dynamical Systems, and an Introduction to Chaos, 3rd edition (Academic Press), 432 pp.

Howard, L. N. \& Krishnamurti, R. K. [1986] "Large-scale flow in turbulent convection: A mathematical model," J. Fluid Mech. 170, 385-410.

Jordan, D. W. \& Smith, P. [2007] Nonlinear Ordinary Differential Equations. An Introduction for Scientists and Engineers, 4th edition (Oxford University Press, NY), 560 pp.

Kreyszig, E. [2011] Advanced Engineering Mathematics, 10th edition (John Wiley \& Sons, Inc.), 1113 pp.

Lorenz, E. [1963] "Deterministic nonperiodic flow," J. Atmos. Sci. 20, 130-141.

Lorenz, E. N. [1969] "The predictability of a flow which possesses many scales of motion," Tellus 21, 289-307.

Lorenz, E. N. [1972] "Predictability: Does the flap of a butterfly's wings in Brazil set off a tornado in Texas?" Proc. 139th Meeting of AAAS Section on Environmental Sciences, New Approaches to Global Weather: GARP (AAAS, Cambridge, MA), 5 pp.

Lorenz, E. N. [1993] The Essence of Chaos (University of Washington Press, Seattle), 227 pp.

Lorenz, E. N. [1996] "Predictability a problem partly solved," Seminar on Predictability, Vol. I (ECMWF).

Lorenz, E. N. [2005] "Designing chaotic models," J. Atmos. Sci. 62, 1574-1587.

Lorenz, E. N. [2008] "The butterfly effect," Premio Felice Pietro Chisesi e Caterina Tomassoni award lecture, University of Rome, Rome.
Moon, S., Han, B.-S., Park, J., Seo, J. M. \& Baik, J.J. [2017] "Periodicity and chaos of high-order Lorenz systems," Int. J. Bifurcation and Chaos 27, 1750176$1-11$.

Musielak, Z. E., Musielak, D. E. \& Kennamer, K. S. [2005] "The onset of chaos in nonlinear dynamical systems determined with a new fractal technique," Fractals 13, 19-31.

Nambu, Y. [1973] "Generalized Hamiltonian dynamics," Phys. Rev. D 7, 2403.

Nevir, P. \& Blender, R. [1994] "Hamiltonian and Nambu representation of the non-dissipative Lorenz equations," Beitraege zur Physik der Atmosphaere 67, 133-140.

Pelino, V., Maimone, F. \& Pasini, A. [2014] "Energy cycle for the Lorenz attractor," Chaos Solit. Fract. 64, 67-77.

Press, W. H., Teukolsky, S. A., Vetterling, W. T. \& Flannery, B. P. [1992] Numerical Recipes in $C$ (Cambridge University Press), 994 pp.

Qi, G. \& Liang, X. [2016] "Force analysis of Qi chaotic system," Int. J. Bifurcation and Chaos 26, 1650237$1-13$.

Qi, G. [2017] "Energy cycle of brushless DC motor chaotic system," Appl. Math. Model. 51, 686-697.

Roupas, Z. [2012] "Phase space geometry and chaotic attractors in dissipative Nambu mechanics," J. Phys. A - Math. Theor. 45, 195101.

Roy, D. \& Musielak, Z. E. [2007a] "Generalized Lorenz models and their routes to chaos. I. Energy-conserving vertical mode truncations," Chaos Solit. Fract. 32, 1038-1052.

Roy, D. \& Musielak, Z. E. [2007b] "Generalized Lorenz models and their routes to chaos. II. Energyconserving horizontal mode truncations," Chaos Solit. Fract. 31, 747-756.

Roy, D. \& Musielak, Z. E. [2007c] "Generalized Lorenz models and their routes to chaos. III. Energyconserving horizontal and vertical mode truncations," Chaos Solit. Fract. 33, 1064-1070.

Saltzman, B. [1962] "Finite amplitude free convection as an initial value problem," J. Atmos. Sci. 19, 329-341.

Shen, B.-W., Atlas, R., Reale, O., Lin, S.-J., Chern, J.D., Chang, J., Henze, C. \& Li, J.-L. [2006] "Hurricane forecasts with a global mesoscale-resolving model: Preliminary results with hurricane Katrina(2005)," Geophys. Res. Lett. 33.

Shen, B.-W., Tao, W.-K. \& Wu, M.-L. [2010] "African easterly waves in 30-day high-resolution global simulations: A case study during the 2006 NAMMA period," Geophys. Res. Lett. 37, L18803.

Shen, B. W., DeMaria, M., Li, J.-L. F. \& Cheung, S. [2013] "Genesis of hurricane sandy (2012) simulated with a global mesoscale model," Geophys. Res. Lett. 40. 
Shen, B.-W. [2014] "Nonlinear feedback in a five-dimensional Lorenz model," J. Atmos. Sci. 71, 1701-1723.

Shen, B.-W. [2015] "Nonlinear feedback in a six-dimensional Lorenz model. Impact of an additional heating term," Nonlin. Process. Geophys. 22, 749-764.

Shen, B.-W . [2016] "Hierarchical scale dependence associated with the extension of the nonlinear feedback loop in a seven-dimensional Lorenz model," Nonlin. Process. Geophys. 23, 189-203.

Shen, B.-W. [2017] "On an extension of the nonlinear feedback loop in a nine-dimensional Lorenz model," Chaot. Model. Simul. (CMSIM) 2, 147-157.

Shen, B.-W. \& Faghih-Naini, S. [2017] "On recurrent solutions in high-dimensional non-dissipative Lorenz models," The 10th Chaos Modeling and Simulation Int. Conf. (CHAOS2017), Barcelona, Spain.

Shen, B.-W. [2018] "On periodic solutions in the nondissipative Lorenz model: The role of the nonlinear feedback loop," Tellus A 70, 1471912.

Shen, B.-W., Pielke Sr., R. A., Zeng, X., FaghihNaini, S., Shie, C.-J., Atlas, R., Baik, J.-J. \& Reyes, T. A. L. [2018] "Butterfly effects of the first and second kinds in Lorenz models," The 11th Chaos Int. Conf. (CHAOS2018), Rome, Italy. Presentation slides are available from Reseachgate: https://doi. org/10.13140/RG.2.2.16647.70564 (under revisions).

Smale, S. [1998] "Mathematical problems for the next century," Math. Intell. 20, 7-15.

Solomon, S., Qin, D., Manning, M., Chen, Z., Marquis, M., Averyt, K., Tignor, M. \& Miller Jr., H. L. (eds.) [2007] Climate Change 200\%: The Physical Science Basis (Cambridge University Press), 996 pp.

Sparrow, C. [1982] The Lorenz Equations: Bifurcations, Chaos, and Strange Attractors, Appl. Math. Sci., Vol. 269 (Springer, NY).
Sprott, J. C. [2003] Chaos and Time-Series Analysis (Oxford University Press), 528 pp. The numerical method is briefly discussed at http://sprott.physics. wisc.edu/chaos/lyapexp.htm.

Stewart, I. [2000] "The Lorenz attractor exists," Nature 406, 948-949.

Strogatz, S. H. [2015] Nonlinear Dynamics and Chaos. With Applications to Physics, Biology, Chemistry, and Engineering (Westview Press, Boulder, CO), 513 pp.

Thiffeault, J.-L. \& Horton, W. [1996] "Energy-conserving truncations for convection with shear flow," Phys. Fluids 8, 1715-1719.

Treve, Y. M. \& Manley, O. P. [1982] "Energy conserving Galerkin approximations for 2-D hydrodynamic and MHD Benard convection," Physica D 4, 319-342.

Tucker, W. [2002] "A rigorous ODE solver and Smale's 14th problem," Found. Comput. Math. 2, 53117.

Wolf, A., Swift, J. B., Swinney, H. L. \& Vastano, J. A. [1985] "Determining Lyapunov exponents from a time series," Physica D 16, 285-317.

\section{Appendix A}

\section{Incommensurate Frequencies Associated with the Extended NFL}

To reveal the role of the NFL in producing oscillatory solutions, below, we analyze the nondissipative 9DLM. After linearizing the model, we can choose $Y_{c}=0, Z_{c}=r,\left(Y_{j}\right)_{c}=0$, and $\left(Z_{j}\right)_{c}=\frac{r}{j+1}$, $j \in[1, N]$. Then, we obtain the following matrix to the linearized system:

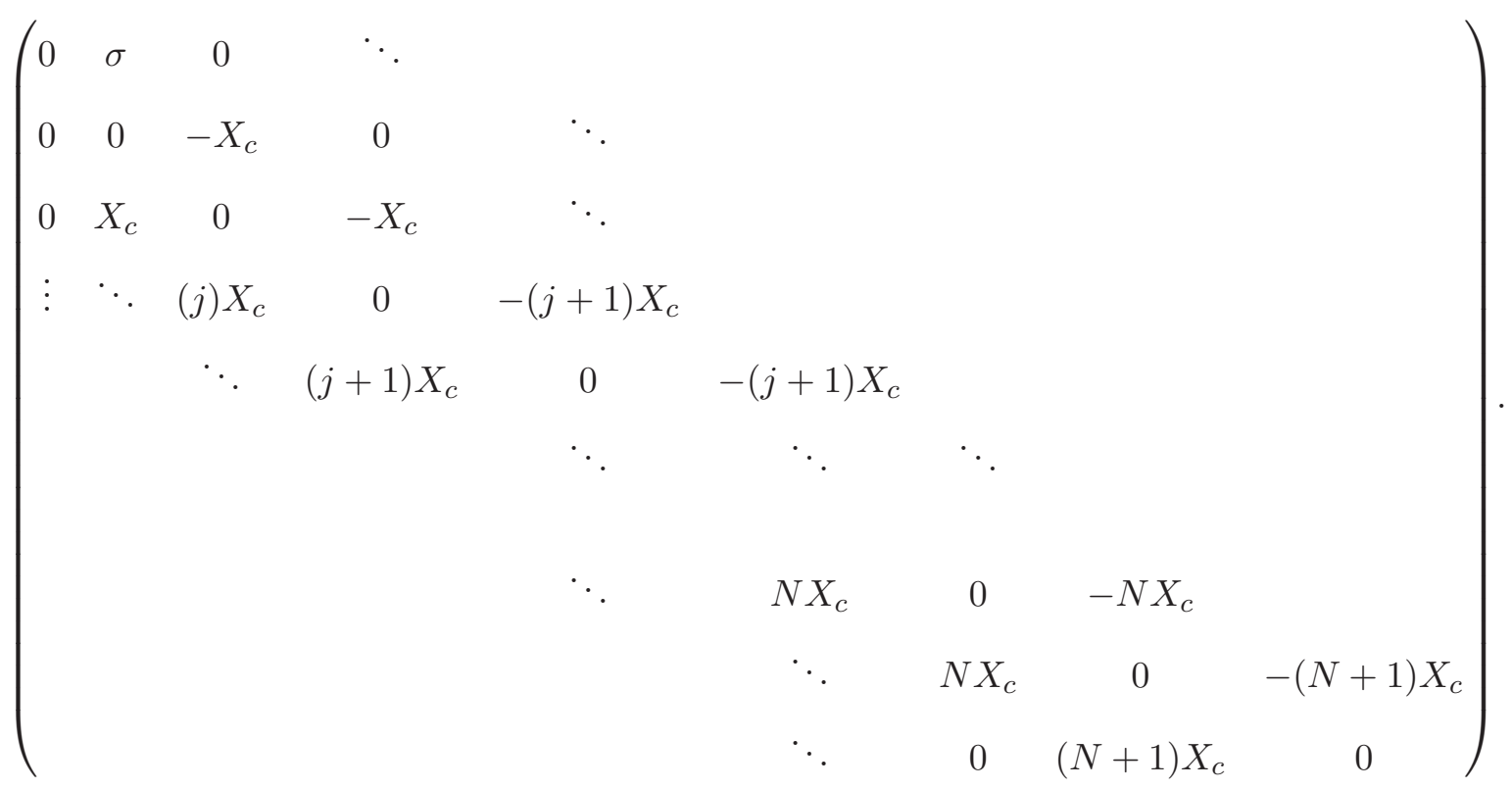


(a)

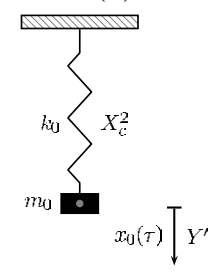

(b)

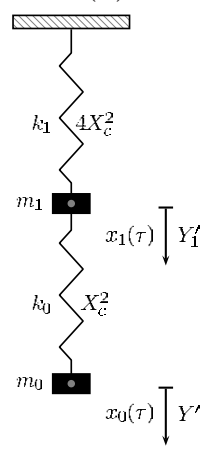

(c)

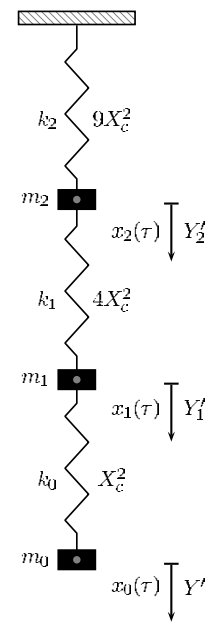

(d)

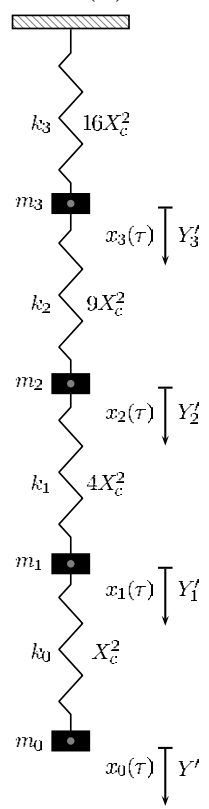

Fig. 11. (a) Systems with one mass and one spring, (b) two masses and two springs, (c) three masses and three springs and (d) four masses and four springs. The four masses are identical (i.e. $m_{0}=m_{1}=m_{2}=m_{3}$ ). Four spring constants, $k_{0}, k_{1}$, $k_{2}$, and $k_{3}$ are selected as $X_{c}^{2}, 4 X_{c}^{2}, 9 X_{c}^{2}$, and $16 X_{c}^{2}$, respectively. The governing equations for the above systems in panels (a)-(d) are identical to those for the locally linear 3D-NLM, 5D-NLM, 7D-NLM, and 9D-NLM, respectively. The comparison illustrates how the nonlinear feedback loop and its extension, enabled by the proper selection of high wavenumber modes, can produce recurrent (i.e. periodic or quasi-periodic) solutions.

All elements in the main diagonal are zeros, indicating no dissipations. Let $i$ be the index for the row, $i \in[1, M]$. For $i>1, j=\operatorname{int}((i-2) / 2)$ and, thus, $j \in[0, N]$ with $N=(M-3) / 2$. Namely, for each $j$, there are two rows in Eq. A.1. The corresponding equations can be reduced and become the following second order ODE:

$$
\begin{aligned}
\frac{d^{2} x_{j}}{d \tau^{2}}= & -k_{j}\left(x_{j}-x_{j+1}\right) \\
& -k_{j-1}\left(x_{j}-x_{j-1}\right), \quad j \in[0, N],
\end{aligned}
$$

here $x_{N+1}=0, x_{-1}=0, x_{j}=Y_{j}, Y_{0}=Y$, $k_{j}=\left((j+1) X_{c}\right)^{2}$, and $k_{-1}=0$. Equation (A.2) is indeed analogous to a system with multiple masses and springs. For example, with $M=5$ in the 5DLM, Eq. (A.2) is identical to a system with two masses and two springs Faghih-Naini \& Shen. 2018: Fay \& Graham, 2003; Krevszig, 2011]. The 9DLM with $M=9$ has $N=3$ and $j \in[0,3]$. The corresponding Eq. (A.2) leads to the following:

$$
\begin{aligned}
& \frac{d^{2} x_{0}}{d \tau^{2}}=-k_{0}\left(x_{0}-x_{1}\right), \\
& \frac{d^{2} x_{1}}{d \tau^{2}}=-k_{1}\left(x_{1}-x_{2}\right)-k_{0}\left(x_{1}-x_{0}\right),
\end{aligned}
$$

$$
\begin{aligned}
& \frac{d^{2} x_{2}}{d \tau^{2}}=-k_{2}\left(x_{2}-x_{3}\right)-k_{1}\left(x_{2}-x_{1}\right), \\
& \frac{d^{2} x_{3}}{d \tau^{2}}=-k_{3} x_{3}-k_{2}\left(x_{3}-x_{2}\right) .
\end{aligned}
$$

As shown in Fig. 11, Eqs. (A.3)-A.6 represent the system with four springs and four masses with the same weight. From top to bottom, $x_{3}(\tau)$, $x_{2}(\tau), x_{1}(\tau)$, and $x_{0}(\tau)$ are the displacements of the centers of four masses $\left(m_{3}, m_{2}, m_{1}\right.$, and $\left.m_{0}\right)$ from equilibrium. The first spring (i.e. the uppermost spring) with a spring constant $k_{3}$ is attached to the ceiling on one end and to the mass, $m_{3}$, on the other end. The upper (lower) end of the second spring with a spring constant $k_{2}$ is attached to mass $m_{3}\left(m_{2}\right)$. The upper (lower) end of the third spring with a spring constant $k_{1}$ is attached to mass $m_{2}$ $\left(m_{1}\right)$. The upper (lower) end of the fourth (the lowest) spring with a spring constant $k_{0}$ is attached to mass $m_{1}\left(m_{0}\right)$. The top three masses experience two restoring forces from two springs while the bottom mass is only influenced by the lowest spring. 